\title{
PUBLIC AND PRIVATE LEARNING FROM PRICES, STRATEGIC SUBSTITUTABILITY AND COMPLEMENTARY, AND EQUILIBRIUM MULTIPLICITY
}

Carolina Manzano

Xavier Vives 
The Public-Private Sector Research Center is a Research Center based at IESE Business School. Its mission is to develop research that analyses the relationships between the private and public sectors primarily in the following areas: regulation and competition, innovation, regional economy and industrial politics and health economics.

Research results are disseminated through publications, conferences and colloquia. These activities are aimed to foster cooperation between the private sector and public administrations, as well as the exchange of ideas and initiatives.

The sponsors of the SP-SP Center are the following:

- Accenture

- Ajuntament de Barcelona

- Caixa Manresa

- Cambra Oficial de Comerç, Indústria i Navegació de Barcelona

- Departament d' Economia i Finances de la Generalitat de Catalunya

- Departament d' Innovació, Universitats i Empresa de la Generalitat de Catalunya

- Diputació de Barcelona

- Endesa

- FOBSIC

- Fundació AGBAR

- Garrigues

- Institut Català de les Indústries Culturals

- Mediapro

- Microsoft

- Sanofi Aventis

- ATM, FGC y TMB

The contents of this publication reflect the conclusions and findings of the individual authors, and not the opinions of the Center's sponsors. 


\title{
Public and private learning from prices, strategic substitutability and complementarity, and equilibrium multiplicity
}

\author{
Carolina Manzano \\ U. Rovira i Virgili*
}

\author{
Xavier Vives \\ IESE Business School and UPF ${ }^{\dagger}$
}

July 2010

\begin{abstract}
We study a general static noisy rational expectations model, where investors have private information about asset payoffs, with common and private components, and about their own exposure to an aggregate risk factor, and derive conditions for existence and uniqueness (or multiplicity) of equilibria. We find that a main driver of the characterization of equilibria is whether the actions of investors are strategic substitutes or complements. This latter property in turn is driven by the strength of a private learning channel from prices, arising from the multidimensional sources of asymmetric information, in relation to the usual public learning channel. When the private learning channel is strong (weak) in relation to the public we have strong (weak) strategic complementarity in actions and potentially multiple (unique) equilibria. The results enable a precise characterization of whether information acquisition decisions are strategic substitutes or complements. We find that the strategic substitutability in information acquisition result obtained in Grossman and Stiglitz (1980) is robust.

JEL Classification: D82, D83, G14

Keywords: Rational expectations equilibrium, strategic complementarity, multiplicity of equilibria, asymmetric information, risk exposure, hedging, supply information.
\end{abstract}

\footnotetext{
* Manzano is grateful for financial support from the Spanish Ministerio de Educación y Ciencia, through the project SEJ2007-67580-C02-01/ECON.

${ }^{\dagger}$ The research in this paper is part of the project Information and Competition, European Advanced Grant (no. 230254) of the European Research Council. Vives is also grateful to project Consolider-Ingenio CSD2006-00016, project ECO2008-05155 of the Spanish Ministry of Education and Science, the Barcelona GSE Research Network for financial support, and the MIT Economics Department for its hospitality.
} 


\section{Introduction}

This paper considers a general noisy rational expectations model of the CARAGaussian class where traders receive both a signal about the asset payoff and a shock to their endowment, both private information. We characterize the linear partially revealing equilibria of the model and show how public and private learning from prices drives the strategic character of the actions (substitutes or complements) of investors. The model unifies and generalizes the models received in the literature.

The presence of multiple equilibria has proved important to explain crises and crashes (e.g., Gennote and Leland (1990)), as well as showing the possibility of strategic complementarity in information acquisition. However, in traditional rational expectations models with asymmetric information (Grossman and Stiglitz (1980), Hellwig (1980), Diamond and Verrecchia (1981), Admati (1985), Vives (1995), see ch. 4 in Vives (2008) for an overview) there exists a unique linear partially revealing rational expectations equilibrium. An exogenously more informative price makes rational investors put less weight on their private signal and generates a response which makes the equilibrium price less informative. ${ }^{1}$ There is aggregate strategic susbtitutability in the actions of informed investors and this implies that the equilibrium is unique. Moreover, stock prices are always more informative when more investors with private information (about the liquidation value) trade. This implies that private information is less valuable, and hence, traders have less incentives to get informed as the fraction of informed traders increases. In short, there is strategic substitutability in information acquisition. In this paper we want to explore the drivers of aggregate strategic susbtitutability or complementarity, and therefore the conditions for multiplicity, as well as the links with the incentives to acquire information.

Our model incorporates two essential features of modern trading in a financial market. First, traders receive typically multidimensional private signals about asset payoffs and aggregate risk factors. For example, the individual exposure of an investor to an aggregate risk factor is typically his private information. The aggregate risk factor may be a stock or derivatives index, a housing price index, or a wage index. A risk averse investor will have an incentive to hedge his exposure to such aggregate risks. The risk factor may be also an aggregate liquidity shock. Second, the aggregate private information of traders about the asset payoff typically does not reveal its liquidation value, there is residual uncertainty. Furthermore, the model we propose avoids the need for noise traders, and relies only on rational traders. Nevertheless, our framework is similar to an economy with private information on noisy supply.

We build a model of the CARA-Gaussian family with these features which has as particular cases the main extant models in the literature. In our economy a trader receives an endowment shock, which is his private information about the aggregate endowment shock. An informed trader receives also a private sig-

\footnotetext{
${ }^{1}$ Vives (1993) explores the dynamic implications of this fact.
} 
nal about the asset payoff with error term which is potentially correlated with the error terms of the signals of other traders. This correlation may arise if private signals are based on a common information source. ${ }^{2}$ With correlated signals the collective information of informed investors does not reveal the asset value, that is, there is residual uncertainty. Furthermore, the signal correlation allows us to parameterize the degree of asymmetric information among informed traders. This generalizes the models in the literature: Grossman and Stiglitz (1980) assume that informed investors observe the same signal about the liquidation value (perfectly correlated private signals errors); in contrast, Diamond and Verrecchia (1981), or Hellwig (1980) assume that private signal errors are uncorrelated. As we will see the assumption of uncorrelation among private signal errors conditions some results. ${ }^{3}$

Our results are as follows. We obtain that when private signal noises among informed traders are correlated the existence of a linear partially revealing rational expectations equilibrium is guaranteed, with the possibility of multiple equilibria (in such a case typically three). Strong strategic complementarity in actions of investors is the driver of potential multiplicity of equilibria. In our model because of the presence of multiple sources of private information there is both a private and a public learning channel from prices (as in Medrano and Vives (2004) and Amador and Weill (2010)). The private learning channel arises because an investor uses his own exposure to the aggregate risk factor to improve his estimate of the asset payoff when looking at the price. Then an increase in the informativeness of the price about the asset payoff generates two effects. First, a trader finds optimal to rely more on the information provided by the private channel. This tends to make investors' actions strategic complements, that is, when other investors' demands rely more on their private information, a trader has more incentives to rely more on his private information. Second, a trader finds optimal to rely more on the improved public information and less on the private information. This classical channel tends to make investors' actions strategic substitutes, when other investors' demands rely more on their private information, a trader has less incentives to rely on his private information. Which of the two effects dominates depends on parameter values. However, for an investor who has no information on the asset payoff strategic complementarity prevails. We obtain that when, on aggregate, actions are either strategic substitutes or moderate strategic complements, the equilibrium is unique. Thus, a necessary condition for multiple equilibria is strong strategic complementarities in actions, if this happens at relevant points then the condition is sufficient.

\footnotetext{
${ }^{2}$ For instance, suppose that the private information signals are based on an internal accounting report (where the information takes the form of the firm's value plus and error). In this case the errors on private information signals will be correlated since all of them include the error in the internal report.

${ }^{3}$ This happens in other models in the literature. In a model with coordination motives and information choice, Hellwig and Veldkamp (2009) show that for the equilibrium to be unique, signal noise across agents must be independent. These authors suggest that this independence assumption should be well-founded because it is stringent and it ultimately determines the predictions of their model.
} 
The forces that push for a low degree of strategic complementarity and uniqueness of equilibrium therefore, are parameter constellations where the private learning channel from prices is weak (and we approach a situation with a unique source of asymmetric information). Those are when the fraction of informed traders about the asset payoff is large and the correlation among the private signal errors is high, or when investors almost do not hold information about the aggregate supply, or when informed investors about the asset payoff have a low risk weighted information advantage.

With correlated signals when strategic complementarity in actions is moderate the equilibrium is unique; when it is strong (at relevant points) then we have three equilibria. Only the middle equilibrium in the multiple equilibria situation displays strategic complementarity in information acquisition. However, it is unstable with respect to adaptive dynamics. In this sense the finding of strategic susbtitutability in information acquisition of Grossman and Stiglitz (1980) is robust. ${ }^{4}$

There is an extensive literature on rational expectations models with asymmetric information. Several extensions of the models proposed by Grossman and Stiglitz (1980) and Diamond and Verrecchia (1981) show the possibility of multiple equilibria ${ }^{5}$ and the possibility of strategic complementarity in information acquisition. Lundholm (1988) extends the rational expectations competitive model of Diamond and Verrecchia (1981), assuming that each investor receives both a public and a private signal. He proves the existence of a symmetric linear rational expectations equilibrium, with the possibility of multiplicity of such equilibria. ${ }^{6}$ Barlevy and Veronesi (2008) show that when fundamentals and noise trading are correlated the existence of multiple equilibria and strategic complementarity in information acquisition may arise. ${ }^{7}$ Ganguli and Yang (2009) consider a variation of the model of Diamond and Verrecchia (1981) where traders observe information about the aggregate supply of the stock and where private signals have uncorrelated error terms. They prove that a linear partially revealing rational expectations equilibrium may not exist. In case of existence (except for a set of parameters of measure zero), there are two of such

\footnotetext{
${ }^{4}$ The only caveat is that there are some parameter constellations such that strategic substitutability in actions is so strong that the unique equilibrium is unstable.

${ }^{5}$ An early analysis of multiplicity is McCafferty and Driskill (1980).

${ }^{6}$ The main focus of his paper is the analysis of some curious comparative statics results. More precisely, Lundholm shows that when public and private signals' errors are positive correlated, the equilibrium price of the risky asset may move inversely with a signal. This contrasts with the intuitive result derived in rational expectations models with one risky asset in which the equilibrium price of the risky security increases in the signals observed by investors (see, for instance, Hellwig (1980) and Diamond and Verrecchia (1981)).

${ }^{7}$ Barlevy and Veronesi (2000) propose a model with risk-neutral traders who face a borrowing constraint, in the presence of noise traders and where the fundamentals follow a binomial distribution. They claim that as more traders acquire private information prices need not become more informative and, consequently, investors may want to acquire more private information. However, Chamley (2008a) proves that the previous paper has a mistake in the expression for the value of information. Barlevy and Veronesi (2008) presents a correction. Other models where strategic complementarities in information acquisition arise are Hirshleifer et al. (1994), Veldkmap (2006, a, b) and Chamley (2007).
} 
equilibria, in one equilibrium the market exhibits strategic complementarity in information acquisition, while in the other there is strategic substitutability. Our results show that the potential non-existence of equilibria in Ganguli and Yang's framework is not robust to small perturbations in the correlation coefficient of private signal errors.

Medrano and Vives (2004), Amador and Weill (2010), and Hatchondo et al. (2010) explore the implications of the private learning channel from prices (respectively, on the welfare analysis of insider trading, on the welfare impact of public information, and on the amplification effect of aggregate exposure on asset prices) when individual exposures to an aggregate risk factor are private information. In our model, as in Amador and Weill (2010), the strength of the private learning channel from prices is the source of strategic complementarity in actions. Goldstein et al. (2010) propose a model where the information revealed in the course of a speculative attack is used by the central bank in its policy decisions. Strategic complementarities among currency speculators arise due to the feedback effect that the information in their trades has on the policy decision of the central bank.

The remainder of this paper is organized as follows. Section 2 outlines the notation and the hypotheses of the model. Section 3 characterizes the symmetric linear equilibria in the general setup and analyzes existence, uniqueness/multiplicity and stability properties of equilibria. Comparative statics and information acquisition incentives are dealt with in Section 4 and some particular cases are analyzed in Section 5. Concluding remarks are presented in Section 6. Finally, proofs are gathered in the Appendix.

\section{The model}

Consider a static asset market model with differential information, where a continuum of risk averse investors exchange a risky asset with liquidation value $v \sim N\left(\bar{v}, \tau_{v}^{-1}\right)$, and a riskless asset with unitary return.

Investor $i \in[0, \mu]$ is (privately) informed about $v$. She has CARA preferences, is endowed with $u_{i}$ shares of the risky asset, and maximizes the expected utility of her wealth: $W_{i}^{I}=v u_{i}+(v-p) x_{i}^{I}$, where $W_{i}^{I}$ represents the trader's $i$ wealth and $x_{i}^{I}$ denotes the units of the risky asset traded by this agent. Thus, $U\left(W_{i}^{I}\right)=-\exp \left\{-W_{i}^{I} / \gamma\right\}$ where $\gamma>0$ is the common degree of risk tolerance. Each informed trader $i$ :

- prior to trading receives a signal $s_{i}=v+\varepsilon_{i}$, where $\varepsilon_{i} \sim N\left(0, \tau_{\varepsilon}^{-1}\right), v$ and $\varepsilon_{i}$ are independent for all $i$ and $\operatorname{cov}\left(\varepsilon_{i}, \varepsilon_{j}\right)=\rho \tau_{\varepsilon}^{-1}$ with $\rho \in[0,1]$ for all $i, j$;

- submits a demand schedule $X^{I}\left(s_{i}, u_{i}, p\right)$, contingent on the private signal $s_{i}$, on the endowment $u_{i}$ and on the price $p$.

Uninformed trader $j \in(\mu, 1]$ has also CARA preferences with the same degree of risk tolerance as informed investors, and maximizes the expected utility 
of his wealth: $W_{j}^{U}=u_{j} v+(v-p) x_{j}^{U}$, where $W_{j}^{U}$ represents the trader's $j$ wealth and $x_{j}^{U}$ denotes the units of the risky asset traded by this agent. Thus, $U\left(W_{j}^{U}\right)=-\exp \left\{-W_{j}^{U} / \gamma\right\}$. Each uninformed trader $j$ :

- submits a demand schedule $X^{U}\left(u_{j}, p\right)$, contingent on the private endowment $u_{j}$ and on $p$.

Finally, assume that

- $u_{h}=u+\eta_{h}$, for all $h \in[0,1]$, where the error terms $\eta_{h}$ are i.i.d., with $\eta_{h} \sim N\left(0, \tau_{\eta}^{-1}\right), u \sim N\left(\bar{u}, \tau_{u}^{-1}\right)$ and $u$ and $\eta_{h}$ are independent of all the other random variables in the model,

- given $u$ the average endowment shock reveals $u$, that is, $\int_{0}^{1} u_{h} d h=u$ a.s. $^{8}$

- given $v$, the average signal satisfies

$$
\frac{1}{\mu} \int_{0}^{\mu} s_{i} d i=v+\widetilde{\varepsilon}
$$

where $\widetilde{\varepsilon}=\frac{1}{\mu} \int_{0}^{\mu} \varepsilon_{h} d h$ is normally distributed with $E[\widetilde{\varepsilon}]=0$ and $\operatorname{var}[\widetilde{\varepsilon}]=$ $\rho \tau_{\epsilon}^{-1}$.

Some remarks on the model are in order:

1. We assume that private signals have correlated errors. This is equivalent to having signals with i.i.d. errors, but with a common shock (like in Grundy and McNichols (1989), for example). ${ }^{9}$ What we accomplish is that the aggregate signal does not reveal $v$ and therefore there is residual uncertainty about the liquidation value. Still another related formulation would be to have i.i.d. errors in the signals, but then have a common shock to the liquidation value that is not observed (as in He and Wang (1995), for example). Our formulation therefore should be considered the general case with $\rho$ parameterizing the common component of the shock in the signal residual uncertainty (from none with $\rho=0$ to a common signal with $\rho=1)$.

2. Our information structure allows for a private learning channel from prices. This is so because $u_{h}$ is a private signal about the aggregate endowment for investor $h$. Although the endowments are independent of $v$, investors will find them useful when predicting $v$ since they allow to disentangle part of the noise in the price coming from the aggregate endowment. In our economy investors have information about factors that are not related to payoffs. In real markets, investors typically have not only information

\footnotetext{
${ }^{8}$ We adopt the convention that the Strong Law of Large Numbers holds (see the thecnical appendix in Vives (2008)).

${ }^{9}$ Indeed, suppose that $s_{i}=v+\widetilde{\varepsilon}+\left(\varepsilon_{i}-\widetilde{\varepsilon}\right)$ where $\widetilde{\varepsilon} \sim N\left(0, \rho \tau_{\varepsilon}^{-1}\right)$ and $\operatorname{cov}\left(\left(\varepsilon_{i}-\widetilde{\varepsilon}\right),\left(\varepsilon_{j}-\widetilde{\varepsilon}\right)\right)=0$ with $\rho \in[0,1]$ for $i \neq j$.
} 
about the payoffs of assets but also about their exposure to some aggregate shock or risk factor. For example, investors may want to hedge their nontradable capital (say human capital) in the stock market. This hedging motive will be stronger the better the correlation of the returns of the non-tradable asset and the stock market. In some other cases traders may obtain information about noise trading or supply shocks. In any case those individual signals or exposure to aggregate risk will constitute private information to the trader and a source which will contribute to multidimensional information in the market (see, for example, Medrano and Vives (2004), Ganguli and Yang (2009), Amador and Weill (2009) and Hatchondo et al. (2010)).

3. Finally, the model we propose avoids the need for noise traders, whose presence has often been criticized in the literature. Nevertheless, our framework is similar to an economy with private information on noisy supply. Concretely, a closely related framework consists in an economy where there are two types of agents: a continuum of rational investors and noise traders. The rational investors are endowed only with a certain amount of the riskless asset and no units of the risky asset. The aggregate supply of the risky asset is driven by noise traders. All the rational investors have private information about the supply of the risky asset and some of them have private information about the liquidation value of the risky asset. The results obtained in this alternative scenario will be similar to ours.

\section{Equilibrium characterization}

We define first a rational expectations equilibrium and consider its implementation in a demand schedule game.

Definition: A rational expectations equilibrium (REE) is a set of trades, contingent on the information that traders have, $\left\{\left(X_{i}^{I}\left(s_{i}, u_{i}, p\right)\right)_{i \in[0, \mu]} ;\left(X_{j}^{U}\left(u_{j}, p\right)\right)_{j \in[\mu, 1]}\right\}$, and a (measurable) price functional $p(v+\widetilde{\varepsilon}, u)$ (i.e., prices measurable in $(v+$ $\widetilde{\varepsilon}, u)$ ) such that

i) markets clear:

$$
\int_{0}^{\mu} X_{i}^{I}\left(s_{i}, u_{i}, p\right) d i+\int_{\mu}^{1} X_{j}^{U}\left(u_{j}, p\right) d j=0 \text { (a.s.); and }
$$

ii) traders in $[0,1]$ optimize: given the price function $p(v+\widetilde{\varepsilon}, u)$,

$$
\begin{gathered}
X_{i}^{I}\left(s_{i}, u_{i}, p\right) \in \underset{x_{i}^{I}}{\arg \max } E\left(U\left(v u_{i}+(v-p) x_{i}^{I}\right) \mid s_{i}, u_{i}, p\right) \text { for } i \in[0, \mu], \text { and } \\
X_{j}^{U}\left(u_{j}, p\right) \in \underset{x_{j}^{U}}{\arg \max } E\left(U\left(v u_{j}+(v-p) x_{j}^{U}\right) \mid u_{j}, p\right) \text { for } j \in(\mu, 1] .
\end{gathered}
$$


Traders understand the relationship between prices and the underlying uncertainty $(v+\widetilde{\varepsilon}, u)$. That is, they conjecture correctly the price function and update their beliefs accordingly. Due to the presence of noise $(u)$, the equilibrium typically will not be fully revealing.

It is well-known (see, for instance, ch. 4 in Vives (2008)) that there is a natural game in demand schedules which implements partially revealing $R E E$. Let traders therefore use demand schedules as strategies. Thus, once each trader has received his private information, investors submit demand schedules contingent on their private information to an auctioneer, who aggregates all the speculators' schedules. Then, the auctioneer finds a market clearing price (as in $(i)$ in the previous definition of $R E E$ ) and, finally, he allocates quantities to satisfy traders' demands.

Denote by $X_{i}^{I}\left(X_{j}^{U}\right)$ a strategy of the informed investor $i$ (uninformed investor $j) \cdot X_{i}^{I}\left(X_{j}^{U}\right)$ is a mapping from the set of her (his) private signals to the set of demand functions. Thus, $X_{i}^{I}\left(s_{i}, u_{i},.\right)$ is a demand function of the informed investor $i$ corresponding to a given private information $\left(s_{i}, u_{i}\right)$. Analogously, $X_{j}^{U}\left(u_{j},.\right)$ is a demand function of the uninformed investor $j$ corresponding to a given private information $u_{j}$.

Let $X^{I}=\left(X_{i}^{I}\right)_{i \in[0, \mu]}$ and $X^{U}=\left(X_{j}^{U}\right)_{j \in(\mu, 1]}$. The market clearing condition implies that

$$
\int_{0}^{\mu} X_{i}^{I}\left(s_{i}, u_{i}, p\right) d i+\int_{\mu}^{1} X_{j}^{U}\left(u_{j}, p\right) d j=0 \text { (a.s.). }
$$

To emphasize the dependence of the market clearing price on the strategies of speculators, we write $p=p\left(X^{I}, X^{U}\right)$. In a Bayesian equilibrium, given his private information, each speculator chooses a demand function to maximize his conditional expected utility, taking as given the strategies of other traders and taking as given the price of the risky asset. We will restrict attention to linear symmetric Bayesian equilibria of the demand schedule game.

Definition: A symmetric linear Bayesian equilibrium (SLE) is a Bayesian equilibrium such that the demand functions are linear and identical, that is,

$$
\begin{aligned}
X_{i}^{I}\left(s_{i}, u_{i}, p\right) & =B_{0}-B_{1} u_{i}-B_{2} p+B_{3} s_{i}, i \in[0, \mu], \text { and } \\
X_{j}^{U}\left(u_{j}, p\right) & =C_{0}-C_{1} u_{j}-C_{2} p, j \in(\mu, 1],
\end{aligned}
$$

where $B_{0}, B_{1}, B_{2}, B_{3}, C_{0}, C_{1}$ and $C_{2}$ are constants.

To solve for a $S L E$ a standard approach is the following. First, positing linear strategies for the traders, we find, using the market clearing condition, an expression for $p$ in terms of $v+\widetilde{\varepsilon}$ and $u$, as follows:

$$
p=p(v+\widetilde{\varepsilon}, u)=A_{0}+A_{1}(v+\widetilde{\varepsilon})-A_{2} u,
$$

where $A_{0}=\frac{\mu B_{0}+(1-\mu) C_{0}}{\mu B_{2}+(1-\mu) C_{2}}, A_{1}=\frac{\mu B_{3}}{\mu B_{2}+(1-\mu) C_{2}}$ and $A_{2}=\frac{\mu B_{1}+(1-\mu) C_{1}}{\mu B_{2}+(1-\mu) C_{2}}$. Second, we compute the asset demands for informed and uninformed types. Third, using (1) we update beliefs about $v$. Finally, we identify coefficients. 
Next, we are interested in deriving under which conditions there exists a $S L E$. In order to perform this, first we express the coefficients of the demand functions in terms of the coefficients of the price function (these expressions are stated in the proof of the Proposition 1). Second, we write the coefficients of the price function as functions of a ratio, $\beta=\frac{A_{1}}{A_{2}}$, and then, we characterize this ratio as a solution of a fixed-point equation. If a solution of this equation exists, then we conclude that there exists a $S L E$. Note that $\beta=\frac{\mu B_{3}}{\mu B_{1}+(1-\mu) C_{1}}$ is

the ratio of the average responsiveness to the private signal on $v$ to the average sensitivity to the endowment shock. That is, it is roughly the ratio of how much information the price reveals about the average signal $v+\widetilde{\varepsilon}$ in relation to how much information about the aggregate shock $u$.

Proposition 1: Let $\beta=\frac{A_{1}}{A_{2}}$. In a SLE, the coefficients of the price function satisfy

$$
\begin{aligned}
& A_{0}=\bar{v}-A_{1} \bar{v}+A_{2} \bar{u}-\frac{1}{\gamma\left(\mu \tau^{I}+(1-\mu) \tau^{U}\right)} \bar{u} \\
& A_{1}=1-\frac{\tau_{v}}{\left(\mu \tau^{I}+(1-\mu) \tau^{U}\right)} \text { and } \\
& A_{2}=\frac{1+\beta \gamma \tau_{u} \tau_{\varepsilon}\left(\frac{\mu(1-\rho)}{\beta^{2} \rho(1-\rho)\left(\tau_{u}+\tau_{\eta}\right)+\tau_{\varepsilon}}+\frac{(1-\mu)}{\beta^{2} \rho\left(\tau_{u}+\tau_{\eta}\right)+\tau_{\varepsilon}}\right)}{\gamma\left(\mu \tau^{I}+(1-\mu) \tau^{U}\right)},
\end{aligned}
$$

where

$$
\begin{aligned}
\tau^{I} & \equiv\left(\operatorname{var}\left(v \mid s_{i}, u_{i}, p\right)\right)^{-1}=\tau_{v}+\frac{\left(\beta^{2}\left(\tau_{u}+\tau_{\eta}\right)(1-\rho)+\tau_{\varepsilon}\right) \tau_{\varepsilon}}{\rho(1-\rho)\left(\tau_{u}+\tau_{\eta}\right) \beta^{2}+\tau_{\varepsilon}} \text { and } \\
\tau^{U} & \equiv\left(\operatorname{var}\left(v \mid u_{j}, p\right)\right)^{-1}=\tau_{v}+\frac{\left(\tau_{u}+\tau_{\eta}\right) \beta^{2} \tau_{\varepsilon}}{\rho\left(\tau_{u}+\tau_{\eta}\right) \beta^{2}+\tau_{\varepsilon}},
\end{aligned}
$$

and $\beta$ is a solution of the following fixed-point equation:

$$
\beta=f(\beta) \equiv \mu \gamma \frac{\left(\tau_{\eta} \beta^{2}(1-\rho)+\tau_{\varepsilon}\right) \tau_{\varepsilon}}{\left(\beta^{2} \rho\left(\tau_{u}+\tau_{\eta}\right)(1-\rho)+\tau_{\varepsilon}\right)}+(1-\mu) \gamma \frac{\beta^{2} \tau_{\eta} \tau_{\varepsilon}}{\tau_{\varepsilon}+\beta^{2} \rho\left(\tau_{u}+\tau_{\eta}\right)} .
$$

Moreover, if $\rho>0$, the existence of a SLE is guaranteed and there are at most three equilibria.

Remark: The result related to the existence of a $S L E$ is trivial. It follows from the fact that $f(0)=\gamma \mu \tau_{\varepsilon} \geq 0$ and $\lim _{\beta \rightarrow \infty} f(\beta)-\beta<0$ whenever $\rho>0$. At a $S L E, \beta \geq 0$. When $\mu \tau_{\varepsilon}=0$, we have that $\beta=0$, otherwise $\beta>0$.

Note that the previous proposition shows that the result of non-existence of a $S L E$ for some parameter configurations derived in Ganguli and Yang (2009), where $\rho=0$, is not robust. A small perturbation in this correlation coefficient guarantees existence of such an equilibrium. 


\subsection{Strategic substitutability and strategic complemen- tarity}

In this subsection we derive the fixed-point equation given in (2) and relate the slope of the function $f(\cdot)$ to the character of the strategic interaction between the investors. In particular, we analyze the relationship between the investors' optimal demands and the information provided by the price.

We start by noting that, provided $\beta>0$, observing the equilibrium price of the risky asset is informationally equivalent to observing

$$
z=v+\widetilde{\varepsilon}-\frac{1}{\beta} u
$$

The endogenous parameter $\beta$ is directly related to the informativeness of the price about the aggregate signal $v+\widetilde{\varepsilon}$.

Consider the informed trader $i$. Recall that her information set is given by $\left\{s_{i}, u_{i}, p\right\}$. This information set is informationally equivalent to the following three signals $\left\{s_{i}, z_{i}, z\right\}: s_{i}=v+\varepsilon_{i}, z_{i}=z+\frac{1}{\beta} u_{i}=v+\widetilde{\varepsilon}+\frac{1}{\beta} \eta_{i}$, and $z$. This is so since the endowment $u_{i}$ helps in extracting information on $v$ in the price (which depends on the aggregate shock $u$ ). The implication is that the price increases investor $i$ 's public information about $v$, through the signal $z$, and it increases investor $i$ 's private information about $v$, through the signal $z_{i}$. We can decompose trader $i$ 's posterior forecast $E\left(v \mid s_{i}, z_{i}, z\right)$ in a (purely) private and a public component (similarly as in Amador and Weill (2010)):

Conditional on her private information, $s_{i}$ and $z_{i}$, and given a fully diffuse prior, trader $i$ forms a private posterior belief that $v$ is normally distributed with mean and precision:

$$
\begin{aligned}
E^{*}\left(v \mid s_{i}, z_{i}\right) & =\frac{\tau_{\varepsilon}}{\tau_{\varepsilon}+\tau_{\eta} \beta^{2}(1-\rho)} s_{i}+\frac{(1-\rho) \beta^{2} \tau_{\eta}}{\tau_{\varepsilon}+\tau_{\eta} \beta^{2}(1-\rho)} z_{i} \text { and } \\
\tau^{* I} & \equiv\left(\operatorname{var}\left(v \mid s_{i}, z_{i}\right)\right)^{-1}=\tau_{\varepsilon}+\frac{\beta^{2} \tau_{\varepsilon} \tau_{\eta}(1-\rho)^{2}}{\tau_{\varepsilon}+\beta^{2} \rho \tau_{\eta}(1-\rho)}
\end{aligned}
$$

Conditional on the public signal $z$, and given the common prior, trader $i$ forms a public posterior belief that $v$ is normally distributed with mean and precision:

$$
\begin{aligned}
E(v \mid z) & =\bar{v}+\frac{\tau_{\varepsilon} \beta^{2} \tau_{u}}{\tau_{v} \tau_{\varepsilon}+\tau_{u} \beta^{2}\left(\tau_{\varepsilon}+\rho \tau_{v}\right)}\left(z-\left(\bar{v}-\frac{1}{\beta} \bar{u}\right)\right) \text { and } \\
\tau & \equiv(\operatorname{var}(v \mid z))^{-1}=\tau_{v}+\frac{\beta^{2} \tau_{u} \tau_{\varepsilon}}{\tau_{\varepsilon}+\beta^{2} \rho \tau_{u}} .
\end{aligned}
$$

Finally, conditional on her information set, the posterior belief of trader $i$ is that $v$ is normally distributed with mean

$$
E\left(v \mid s_{i}, z_{i}, z\right)=\omega^{I} E^{*}\left(v \mid s_{i}, z_{i}\right)+\left(1-\omega^{I}-\varkappa^{I}\right) E(v \mid z)+\varkappa^{I} \bar{v}
$$


where

$$
\begin{aligned}
\omega^{I} & \equiv \frac{\tau_{\varepsilon}\left(\tau_{\varepsilon}+\tau_{\eta} \beta^{2}(1-\rho)\right)}{\tau_{\varepsilon}\left(\tau_{v}+\tau_{\varepsilon}\right)+\beta^{2}\left(\tau_{u}+\tau_{\eta}\right)(1-\rho)\left(\tau_{\varepsilon}+\rho \tau_{v}\right)} \text { and } \\
\varkappa^{I} & \equiv \frac{\rho \tau_{v}\left(\tau_{\varepsilon}+\beta^{2} \tau_{\eta}(1-\rho)\right)}{\tau_{\varepsilon}\left(\tau_{v}+\tau_{\varepsilon}\right)+\beta^{2}\left(\tau_{u}+\tau_{\eta}\right)(1-\rho)\left(\tau_{\varepsilon}+\rho \tau_{v}\right)},
\end{aligned}
$$

and precision

$$
\tau^{I} \equiv\left(\operatorname{var}\left(v \mid s_{i}, z_{i}, z\right)\right)^{-1}=\frac{\tau_{\varepsilon}\left(\tau_{v}+\tau_{\varepsilon}\right)+\beta^{2}\left(\tau_{u}+\tau_{\eta}\right)(1-\rho)\left(\tau_{\varepsilon}+\rho \tau_{v}\right)}{\tau_{\varepsilon}+(1-\rho)\left(\tau_{u}+\tau_{\eta}\right) \rho \beta^{2}} .
$$

Note that when $\rho=0$ we have that $\varkappa^{I}=0$, and when $\rho=1$ we have that $1-\omega^{I}-\varkappa^{I}=0$ (then only $s_{i}$ is relevant for an informed agent, $E^{*}\left(v \mid s_{i}, z_{i}\right)=s_{i}$ and $\left.E\left(v \mid s_{i}, z_{i}, z\right)=E\left(v \mid s_{i}\right)=\frac{\tau_{\varepsilon}}{\tau_{v}+\tau_{\varepsilon}} s_{i}+\frac{\tau_{v}}{\left(\tau_{v}+\tau_{\varepsilon}\right)} \bar{v}\right)$. In both cases signals are conditionally independent (in the first case about $v$ and in the second case about $v+\widetilde{\varepsilon})$.

Now, consider the uninformed trader $j$. His information set $\left\{u_{j}, p\right\}$ is informationally equivalent to the following two signals: $z_{j}=z+\frac{1}{\beta} u_{j}=v+\widetilde{\varepsilon}+\frac{1}{\beta} \eta_{j}$ and $z$. Performing a similar decomposition for trader $j$ 's posterior belief, we get

$$
E\left(v \mid z_{j}, z\right)=\omega^{U} E^{*}\left(v \mid z_{j}\right)+\left(1-\omega^{U}-\varkappa^{U}\right) E(v \mid z)+\varkappa^{U} \bar{v}
$$

where $\omega^{U} \equiv \frac{\beta^{2} \tau_{\varepsilon} \tau_{\eta}}{\tau_{v} \tau_{\varepsilon}+\beta^{2}\left(\tau_{u}+\tau_{\eta}\right)\left(\tau_{\varepsilon}+\rho \tau_{v}\right)}$ and $\varkappa^{U} \equiv \frac{\beta^{2} \rho \tau_{v} \tau_{\eta}}{\tau_{v} \tau_{\varepsilon}+\beta^{2}\left(\tau_{u}+\tau_{\eta}\right)\left(\tau_{\varepsilon}+\rho \tau_{v}\right)}$,

$$
\begin{aligned}
E^{*}\left(v \mid z_{j}\right) & =z_{j}, \\
\tau^{U} & \equiv\left(\operatorname{var}\left(v \mid z_{j}, z\right)\right)^{-1}=\frac{\tau_{v} \tau_{\varepsilon}+\beta^{2}\left(\tau_{u}+\tau_{\eta}\right)\left(\tau_{\varepsilon}+\rho \tau_{v}\right)}{\tau_{\varepsilon}+\beta^{2} \rho\left(\tau_{u}+\tau_{\eta}\right)}, \text { and } \\
\tau^{* U} & \equiv\left(\operatorname{var}\left(v \mid z_{j}\right)\right)^{-1}=\frac{\beta^{2} \tau_{\varepsilon} \tau_{\eta}}{\tau_{\varepsilon}+\beta^{2} \rho \tau_{\eta}} .
\end{aligned}
$$

Next, using the previous decompositions, we derive an expression for the equilibrium price. Taking into account the transformations of the traders' information sets, the investors' optimal demands can be rewritten as:

$$
\begin{aligned}
x_{i}^{I} & =\gamma \tau^{I}\left(E\left(v \mid s_{i}, z_{i}, z\right)-p-\frac{1}{\gamma \tau^{I}} u_{i}\right), \text { for all } i \in[0, \mu] \text { and } \\
x_{j}^{U} & =\gamma \tau^{U}\left(E\left(v \mid z_{j}, z\right)-p-\frac{1}{\gamma \tau^{U}} u_{j}\right), \text { for all } j \in(\mu, 1] .
\end{aligned}
$$

Hence, the aggregate demand is given by

$$
\int_{0}^{\mu} \gamma \tau^{I}\left(E\left(v \mid s_{i}, z_{i}, z\right)-p-\frac{1}{\gamma \tau^{I}} u_{i}\right) d i+\int_{\mu}^{1} \gamma \tau^{U}\left(E\left(v \mid z_{j}, z\right)-p-\frac{1}{\gamma \tau^{U}} u_{j}\right) d j
$$


or equivalently,

$$
\gamma \tau^{I} \int_{0}^{\mu} E\left(v \mid s_{i}, z_{i}, z\right) d i+\gamma \tau^{U} \int_{\mu}^{1} E\left(v \mid z_{j}, z\right) d j-u-\gamma\left(\mu \tau^{I}+(1-\mu) \tau^{U}\right) p
$$

Therefore, the market clearing condition implies that

$$
p=\frac{\tau^{I} \int_{0}^{\mu} E\left(v \mid s_{i}, z_{i}, z\right) d i+\tau^{U} \int_{\mu}^{1} E\left(v \mid z_{j}, z\right) d j-\frac{1}{\gamma} u}{\mu \tau^{I}+(1-\mu) \tau^{U}}
$$

By virtue of (4) and (5), it follows that

$$
\int_{0}^{\mu} E\left(v \mid s_{i}, z_{i}, z\right) d i=\omega^{I} \mu(v+\widetilde{\varepsilon})+\mu\left(1-\omega^{I}-\varkappa^{I}\right) E(v \mid z)+\mu \varkappa^{I} \bar{v}
$$

and

$$
\int_{\mu}^{1} E\left(v \mid z_{j}, z\right) d j=\omega^{U}(1-\mu)(v+\widetilde{\varepsilon})+(1-\mu)\left(1-\omega^{U}-\varkappa^{U}\right) E(v \mid z)+(1-\mu) \varkappa^{U} \bar{v}
$$

Substituting these expressions in (7) and taking into account that $E(v \mid z)$ is a linear function of $p$, we get that observing $p$ is equivalent to observing

$$
v+\widetilde{\varepsilon}-\frac{1}{\gamma\left(\mu \tau^{I} \omega^{I}+(1-\mu) \tau^{U} \omega^{U}\right)} u
$$

Comparing this formula with the expression of $z$ stated in (3), we have that, in equilibrium

$$
\beta=f(\beta) \equiv \mu \Omega^{I}+(1-\mu) \Omega^{U}
$$

where $\Omega^{I}=\gamma \tau^{I} \omega^{I}$ and $\Omega^{U}=\gamma \tau^{U} \omega^{U}$. Taking into account the expression of aggregate demand given in (6) and the decompositions of investors' beliefs given in (4) and (5), Expression (8) shows that $\beta$ is a measure of sensibility of the aggregate demand in the investors' private information. Notice that if all private forecasts increase in one unit, the aggregate demand rises $\beta$.

Substituting the expressions of $\Omega^{I}$ and $\Omega^{U}$ in (8), using the expressions of $\tau^{* I}, \tau$, and $\tau^{* U}$, we get the fixed-point-equation stated in Proposition 1 . The function $f(\cdot)$ can be understood as an aggregate (average) best response function which yields the price informativeness, $\beta$ for short, out of the responses of traders to changes to initial price informativeness. There is aggregate strategic susbtitutability (complementarity) when $f^{\prime}(\cdot)<0\left(f^{\prime}(\cdot)>0\right)$ and a change in $\beta$ induces responses which decrease (increase) price informativeness. Strategic susbtitutability is the traditional case in the Grossman-Stiglitz style models.

Consider an informed investor. Using the expression of $\omega^{I}, \tau^{I}, \tau^{* I}$ and $\tau$, we get

$$
\Omega^{I}=H\left(\tau^{* I}, \tau\right)=\gamma \frac{\tau^{* I}}{\frac{\tau_{\varepsilon}^{2}+\tau^{* I} \rho^{2}\left(\tau_{v}-\tau\right)}{\tau_{\varepsilon}\left(\tau_{\varepsilon}+\rho\left(\tau_{v}-\tau\right)\right)}}
$$


Notice that when $\beta$ increases, that is, when other investors' demands rely more on their private information, prices are more informative about $v$. This effect rises both the precision of public information $\tau$ and the precision of private information $\tau^{* I}$. These increases have, in general, two opposite effects on the term $\Omega^{I}$ :

1) The increase in the precision of public information tends to decrease $\Omega^{I}$, which means that an informed trader finds optimal to rely more on the improved public information and less on the private information. Thus, the endogenous public signal generated by prices tends to make investors' decisions strategic substitutes, when other investors' demands rely more on their private information, an informed trader has less incentives to take her private information into consideration. This is the usual effect in the Grossman-Stiglitz model.

2) The increase in the precision of private information tends to increase $\Omega^{I}$, which means that an informed trader finds optimal to rely more on this improved private information. Thus, the endogenous private signal generated by prices tends to make investors' decisions strategic complements, that is, when other investors' demands rely more on their private information, an informed trader has more incentives to take her private information into consideration. This effect arises out of the private learning channel of prices.

Which of the two effects dominates will depend on parameter values. Specifically, we obtain

$$
\frac{\partial \Omega^{I}}{\partial \beta}=\gamma 2 \frac{\left(\tau_{\eta}-\rho\left(\tau_{u}+\tau_{\eta}\right)\right)(1-\rho) \beta \tau_{\varepsilon}^{2}}{\left(\beta^{2} \rho\left(\tau_{u}+\tau_{\eta}\right)(1-\rho)+\tau_{\varepsilon}\right)^{2}} .
$$

Hence, $\frac{\partial \Omega^{I}}{\partial \beta}=0$ if $\rho=1$ and $\frac{\partial \Omega^{I}}{\partial \beta}>0$ if and only if $\rho<\Psi$, where $\Psi \equiv \frac{\tau_{\eta}}{\tau_{u}+\tau_{\eta}}$ is the (squared) correlation coefficient between $u$ and $u_{i}$. When $\Psi$ is large in relation to the the correlation of private signal errors, the private learning channel from prices dominates for informed investors (and $\frac{\partial \Omega^{I}}{\partial \beta}>0$ ).

The intuition for these results is as follows: When $\rho=1$, the price does not provide any additional information in the prediction of $v$ to informed traders, and consequently, the individual only takes into account $s_{i}$. Hence, $\Omega^{I}$, the sensibility of an informed trader's demand to her private information is independent of $\beta$. By contrast, whenever $\rho<1$, the price provides extra information when predicting $v$. If $\rho$ is very large or if $\tau_{\eta}$ is very low, the expression for $\tau^{* I}$ tells us that $\tau^{* I}$ increases with $\beta$, but varies very little. This implies that the first effect dominates, and hence, $\frac{\partial \Omega^{I}}{\partial \beta}<0$. Otherwise, if $\rho$ is low or $\tau_{\eta}$ is large, then the change of $\tau^{* I}$ due to an increase of beta is significative and this makes the second effect to dominate, which implies $\frac{\partial \Omega^{I}}{\partial \beta}>0$. Thus, for informed investors, the private learning channel from prices is important if the (squared) correlation coefficient between $u$ and $u_{i}, \frac{\tau_{\eta}}{\tau_{u}+\tau_{\eta}}$, is large in relation to the correlation of private signal errors.

A similar reasoning may be done for an uninformed trader since in this case 
we get an identical expression for $\Omega^{U}$,

$$
\Omega^{U}=H\left(\tau^{* U}, \tau\right)=\gamma \frac{\tau^{* U}}{\frac{\tau_{\varepsilon}^{2}+\tau^{* U} \rho^{2}\left(\tau_{v}-\tau\right)}{\tau_{\varepsilon}\left(\tau_{\varepsilon}+\rho\left(\tau_{v}-\tau\right)\right)}} .
$$

Again an increase in $\beta$ rises both the precision of public information $\tau$ and the precision of private information $\tau^{* I}$. In general, the first effect tends to decrease $\Omega^{U}$ and the second effect tends to increase $\Omega^{U}$. However, the overall effect of $\beta$ on $\Omega^{U}$ is unambiguous:

$$
\frac{\partial \Omega^{U}}{\partial \beta}=\gamma \frac{2 \tau_{\eta} \tau_{\varepsilon}^{2} \beta}{\left(\tau_{\varepsilon}+\beta^{2} \rho\left(\tau_{u}+\tau_{\eta}\right)\right)^{2}} \geq 0
$$

indicating that for the uninformed traders the second effect always prevails. Note that even if the value of $\tau_{\eta}$ is low, we have that $\frac{\partial \Omega^{U}}{\partial \beta}>0$. The reason is that when $\tau_{\eta}$ is low, the second effect is low since $\tau^{* U}$ varies little with respect to $\beta$, but the first effect is also low since $\Omega^{U}$ varies little with respect to $\tau$, given that in this case $\tau^{* U}$ is low.

Comparative statics of $f^{\prime}(\beta)$ allows us to determine which parameters amplify/narrow potential strategic complementarities/substitutabilities in the actions of investors. We obtain:

$$
f^{\prime}(\beta)=\mu \frac{2 \gamma\left(\tau_{\eta}-\rho\left(\tau_{u}+\tau_{\eta}\right)\right)(1-\rho) \beta \tau_{\varepsilon}^{2}}{\left(\beta^{2} \rho\left(\tau_{u}+\tau_{\eta}\right)(1-\rho)+\tau_{\varepsilon}\right)^{2}}+(1-\mu) \frac{2 \gamma \tau_{\eta} \tau_{\varepsilon}^{2} \beta}{\left(\tau_{\varepsilon}+\beta^{2} \rho\left(\tau_{u}+\tau_{\eta}\right)\right)^{2}} .
$$

Note that if $\mu=1, \operatorname{sign}\left(f^{\prime}(\beta)\right)=\operatorname{sign}((\Psi-\rho)(1-\rho))$, where $\Psi=\frac{\tau_{\eta}}{\tau_{u}+\tau_{\eta}}$. For $\rho<1$ we have that $f^{\prime}(\cdot)>0$ if and only if $\Psi>\rho$. Straightforward computations yield the following result:

Corollary 2: a) If $\rho \neq 0$, then $\frac{\partial}{\partial \mu} f^{\prime}(\beta)<0$. If $\rho=0$, then $\frac{\partial}{\partial \mu} f^{\prime}(\beta)=0$.

b) $\operatorname{sign}\left(\frac{\partial}{\partial \gamma} f^{\prime}(\beta)\right)=\operatorname{sign}\left(f^{\prime}(\beta)\right)$.

c) If $\tau_{\eta}-\rho\left(\tau_{u}+\tau_{\eta}\right) \geq 0$ (that is, when informed traders' actions are strategic complements), then $\frac{\partial}{\partial \rho} f^{\prime}(\beta) \leq 0, \frac{\partial}{\partial \tau_{\varepsilon}} f^{\prime}(\beta) \geq 0$ and $\frac{\partial}{\partial \tau_{u}} f^{\prime}(\beta) \leq 0$.

The result in a) is due to the fact that it always holds that $\frac{\partial \Omega^{I}}{\partial \beta} \leq \frac{\partial \Omega^{U}}{\partial \beta}$. The result in b) follows directly from the expressions of $\Omega^{I}=\gamma \tau^{I} \omega^{I}$ and $\Omega^{U}=\gamma \tau^{U} \omega^{U}$. The second part of this corollary tells us that a change in the risk tolerance coefficient amplifies/narrows the potential strategic complementarity/substitutability in actions.

\subsection{Uniqueness/multiplicity of equilibrium}

In this subsection we derive sufficient conditions for the uniqueness of SLE in the general setup. In other words, we obtain sufficient conditions for the graph of function $f(\cdot)$ and the 45 -degree line to intersect once. Note that when we have 
(aggregate) strategic susbtitutability -the function $f(\cdot)$ is decreasing $\left(f^{\prime}(\cdot)<0\right)$ or when (aggregate) strategic complementarity is moderate -the 45-degree line is steeper than the function $f(\cdot)\left(f^{\prime}(\cdot)<1\right)$-, the uniqueness of equilibrium is guaranteed. The following corollary provides the parameter configurations for these two cases:

Proposition 3: a) If $(1-\mu) \leq \mu\left(\rho \Psi^{-1}-1\right)(1-\rho)$, then $f^{\prime}(\cdot)<0$ and there exists a unique SLE.

b) If $\frac{27}{64} \gamma^{2} \tau_{\eta} \tau_{\varepsilon} \Psi<\rho$, then $f^{\prime}(\cdot)<1$ and there exists a unique SLE.

When the condition in a) holds, informed traders' actions are strategic substitutes $\left(\frac{\partial \Omega^{I}}{\partial \beta}<0\right)$ and the fraction of uninformed traders is small enough. Taking into account that $f^{\prime}(\beta)=\mu \frac{\partial \Omega^{I}}{\partial \beta}+(1-\mu) \frac{\partial \Omega^{U}}{\partial \beta}$, it follows that $f^{\prime}(\beta)<$ 0 for all $\beta$, leading to a unique equilibrium. In relation to $b$ ) note that if $\frac{27}{64} \gamma^{2} \tau_{\eta} \tau_{\varepsilon} \Psi<\rho$, then $\frac{\partial \Omega^{U}}{\partial \beta}<1$. As $\frac{\partial \Omega^{I}}{\partial \beta} \leq \frac{\partial \Omega^{U}}{\partial \beta}$ always holds, we get that $f^{\prime}(\beta)=\mu \frac{\partial \Omega^{I}}{\partial \beta}+(1-\mu) \frac{\partial \Omega^{U}}{\partial \beta}<1$ for all $\beta$, which warrants uniqueness of equilibrium.

From the corollary it emerges that the forces for strategic substitutability, and uniqueness of equilibrium therefore, are parameter constellations where the private learning channel for prices is weak (and we approach a situation with a unique source of asymmetric/diverse information). Those are when the fraction of informed traders is large ( $\mu$ high) and the correlation among the private signal errors is high ( $\rho$ high), or when investors almost do not hold information about the aggregate supply ( $\Psi$ or $\tau_{\eta}$ low). The same forces together when informed investors have a low risk weighted information advantage about $v\left(\gamma \tau_{\varepsilon}\right.$ low) push for low strategic complementarity.

It is important to remark that all these results allows us to conclude that a necessary condition for multiple equilibria is the presence of strong strategic complementarities in actions at relevant points. However, the derivation of sufficient conditions on the primitives that guarantee multiplicity of equilibria in this general setup is not easy. In Section 5, we study particular cases and, then, necessary and sufficient for multiplicity of equilibria are obtained.

\subsection{Stability}

Until now, we have shown that generically a $S L E$ exists, with the possibility of multiple equilibria. As usual in these cases, the natural question is whether there exists a plausible selection device which implies that traders coordinate on a specific equilibrium. A standard criteria for selecting among equilibria is stability. ${ }^{10}$

Consider that the economy is in equilibrium. Thus, $\beta=f(\beta)$. Suppose that there is a small perturbation in $\beta$, such that the price is informationally

\footnotetext{
${ }^{10}$ There is a long literature on learning and stability of $R E E$ (see Blume, Bray and Easley (1982) and Section 7.1 in Vives (2008) for an introduction).
} 
equivalent to $v+\widetilde{\varepsilon}-\frac{1}{\beta^{\prime}} u$. Then, the agents modify their demands and, in particular, their weights on private information $\Omega^{I}\left(\beta^{\prime}\right)$ and $\Omega^{U}\left(\beta^{\prime}\right)$, for the informed traders and uninformed traders respectively. The aggregate weight becomes $f\left(\beta^{\prime}\right)=\mu \Omega^{I}\left(\beta^{\prime}\right)+(1-\mu) \Omega^{U}\left(\beta^{\prime}\right)$. The question is if the economy will go back to the original $\beta$ or not according to best reply dynamics with the aggregate best response function $f(\cdot) .{ }^{11}$ This leads to the following definition:

Definition: A SLE is stable (unstable) if and only if its corresponding value for $\beta$ is a stable (unstable) fixed point for the best response function $f(\cdot)$ (i.e., if and only if its corresponding value for $\beta$ satisfies $\left.\left|f^{\prime}(\beta)\right|<1\right)$.

Note that if $f^{\prime}(\cdot)>0$ and there are three (regular) equilibria (that is, for which $\left.f^{\prime}(\beta) \neq 1\right)$ then necessarily the extremal ones are stable and the middle one unstable. If $f^{\prime}(\cdot)<0$ the equilibrium will be unique but may be unstable if strategic susbtitutability is strong enough (and $\left|f^{\prime}(\beta)\right|>1$ ).

\section{Comparative statics and information acquisi- tion}

We derive some comparative statics results and apply them to analyze the incentives to acquire information about the asset payoff.

\subsection{Some comparative statics}

We now analyze how some parameters affect the information that the price reveals about the liquidation value and about the aggregate supply. Using the expression of the equilibrium price, we get that the informational content of the price about the liquidation value and the aggregate supply is given by

$$
(\operatorname{var}(v \mid p))^{-1}=\tau_{v}+\frac{\beta^{2} \tau_{u} \tau_{\varepsilon}}{\tau_{\varepsilon}+\beta^{2} \rho \tau_{u}} \text { and }(\operatorname{var}(u \mid p))^{-1}=\tau_{u}+\frac{\tau_{\varepsilon} \tau_{v}}{\left(\tau_{\varepsilon}+\rho \tau_{v}\right) \beta^{2}} .
$$

Remark: As $\beta$ is independent of $\tau_{v}$, the previous expressions tell us that $\frac{\partial}{\partial \tau_{v}}(\operatorname{var}(v \mid p))^{-1}>0$ and $\frac{\partial}{\partial \tau_{v}}(\operatorname{var}(u \mid p))^{-1}>0$ in any equilibrium.

Next, we analyze the effect of a change in the risk tolerance coefficient and in the fraction of informed traders. Using the chain rule for a parameter $\varsigma$ :

$$
\begin{aligned}
\frac{\partial}{\partial \varsigma}(\operatorname{var}(v \mid p))^{-1} & =\frac{\partial}{\partial \beta}(\operatorname{var}(v \mid p))^{-1} \frac{\partial \beta}{\partial \varsigma}, \text { and } \\
\frac{\partial}{\partial \varsigma}(\operatorname{var}(u \mid p))^{-1} & =\frac{\partial}{\partial \beta}(\operatorname{var}(u \mid p))^{-1} \frac{\partial \beta}{\partial \varsigma} .
\end{aligned}
$$

\footnotetext{
${ }^{11}$ This definition is similar to the one used by Chamley (2008b) when studying the stability of equlibria in Ganguli and Yang (2009).
} 
Since $\frac{\partial}{\partial \beta}(\operatorname{var}(v \mid p))^{-1}>0$ and $\frac{\partial}{\partial \beta}(\operatorname{var}(u \mid p))^{-1}<0$, we obtain:

$$
\begin{aligned}
\operatorname{sign}\left(\frac{\partial}{\partial \varsigma}(\operatorname{var}(v \mid p))^{-1}\right) & =\operatorname{sign}\left(\frac{\partial \beta}{\partial \varsigma}\right) \text { and } \\
\left.\operatorname{sign}\left(\frac{\partial}{\partial \varsigma}(\operatorname{var}(u \mid p))\right)^{-1}\right) & =-\operatorname{sign}\left(\frac{\partial \beta}{\partial \varsigma}\right), \text { for } \varsigma=\gamma \text { and } \mu .
\end{aligned}
$$

In order to obtain how $\beta$ changes with respect to $\varsigma=\gamma$ and $\mu$ we have to see how $f(\cdot)$ moves with $\gamma$ and $\mu$. It is easy to see that $f(\cdot)$ is strictly increasing in $\gamma$ and $\mu$, that is, ceteris paribus, more risk tolerance or more informed traders make the aggregate demand to be more sensible to private information (note that the weight that informed agents put on private information is higher than the one put by uninformed ones). It follows that for regular equilibria (for which $\left.f^{\prime}(\beta) \neq 1\right)$, $\operatorname{sign}\left(\frac{\partial \beta}{\partial \varsigma}\right)=\operatorname{sign}\left(1-f^{\prime}(\beta)\right)$. It follows that for any regular equilibrium increasing $\gamma$ or $\mu$ will increase $\beta$ except if at the equilibrium $f^{\prime}(\beta)>$ 1 (in which case $\beta$ decreases). This means that for any regular equilibrium with $f^{\prime}(\beta)<1$ we have that increasing $\gamma$ or $\mu$ increases the informativeness of the price about the asset value in relation to the aggregate endowment shock. When $f^{\prime}(\beta)>1$, the opposite happens.

If $\rho>0$ there are generically either one or three regular equilibria. If $\rho=0$ we know that there are generically two regular equilibria when they exist, and the equilibrium with the highest $\beta$ is unstable (Ganguli and Yang (2009)).

This allows us to obtain the following result:

Proposition 4: In a regular equilibrium with $f^{\prime}(\beta)<1, \quad(\operatorname{var}(v \mid p))^{-1}$ strictly increases with both $\gamma$ and $\mu$ and $(\operatorname{var}(u \mid p))^{-1}$ strictly decreases with both $\gamma$ and $\mu$. If $f^{\prime}(\beta)>1$, results are reversed.

Note that if we are on the middle unstable equilibrium of three equilibria and $\gamma$ or $\mu$ increase adaptive dynamics will converge to the higher equilibrium, increasing $\beta$ (and this will happen even if the unstable equilibrium disappears, see Figure 1 and see the movement of $f(\cdot)$ from the middle to the upper branch increasing $\gamma$ ). When $\rho=0$ and if we are on the high unstable equilibrium, if $\gamma$ or $\mu$ increases, adaptive dynamics will diverge. 


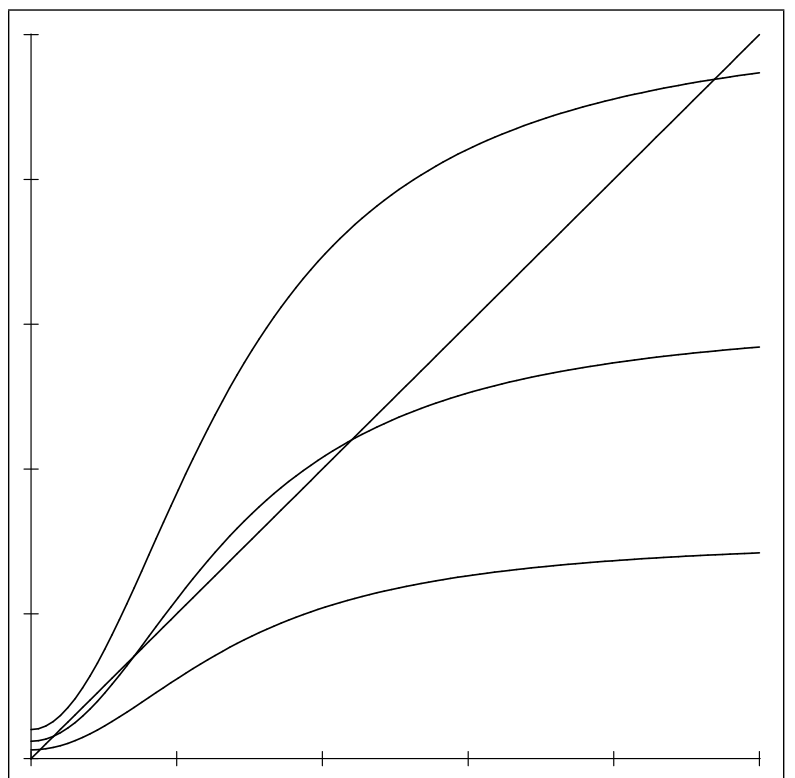

Figure 1. The function $f(\cdot)$ for $\gamma=\frac{3}{2}, \gamma=3$ and $\gamma=5$ (and $\rho=1, \tau_{\eta}=1$,

$$
\left.\tau_{u}=1, \mu=\frac{1}{50} \text { and } \tau_{\varepsilon}=1\right) \text {. }
$$

When $\rho>0$, if we are in a critical equilibrium and if $\gamma$ or $\mu$ increase then the critical equilibrium will disappear and adaptive dynamics will settle on a higher equilibrium (see Figure 2 where the situation is illustrated by increasing $\gamma$ from $\gamma=2.3134$ to 3 ). 


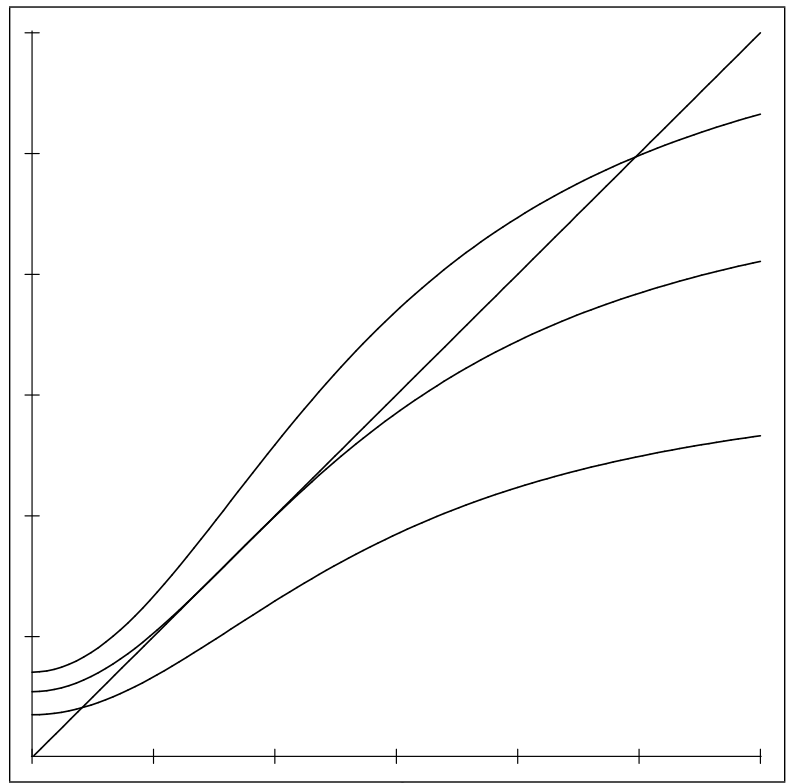

Figure 2. The function $f(\cdot)$ for $\gamma=\frac{3}{2}, \gamma=2.3134$ and $\gamma=3$ (and $\rho=1$,

$$
\left.\tau_{\eta}=1, \tau_{u}=1, \mu=\frac{1}{17} \text { and } \tau_{\varepsilon}=1\right) \text {. }
$$

We see therefore that unambiguous comparative statics results can be derived even for critical and unstable equilibria (see Vives (1990) and Echenique (2002) for general results).

\subsection{Information acquisition incentives}

Next, we analyze the possibility of strategic complementarity in information acquisition. Strategic complementarity (substitutability) in information acquisition means that traders have more (less) incentives to get informed as the fraction of informed traders increases. Formally, let $R(\mu)=\frac{E\left(U\left(W_{i}^{I}\right)\right)}{E\left(U\left(W_{j}^{U}\right)\right)}$, where $E\left(U\left(W_{i}^{I}\right)\right)$ and $E\left(U\left(W_{j}^{U}\right)\right)$ denote the ex-ante expected indirect utility of an informed trader and an uninformed trader, respectively, gross of information costs.

Definition: A market exhibits strategic complementarity (substitutability) in information acquisition if $R^{\prime}(\mu)<0\left(R^{\prime}(\mu)>0\right)$.

Corollary 5: $R(\mu)=\left(\frac{\tau^{U}}{\tau^{I}}\right)^{1 / 2}$.

This result tells us that, similar to Grossman and Stiglitz (1980), the value of information about the liquidation value of the risky asset is related to the square root of the ratio of the precision of uninformed traders to the precision of informed traders. It is easy to see from the expressions for $\tau^{U}$ and $\tau^{I}$ that $\operatorname{sign}\left(R^{\prime}(\mu)\right)=\operatorname{sign}\left(\beta^{\prime}(\mu)\right)$. That is, we have strategic substitutability or 
strategic complementarity in information acquisition depending on whether an increase in the proportion of informed increases or decreases the informativeness of price (in relation to $v+\widetilde{\varepsilon}$ ). When $\beta^{\prime}(\mu)>0\left(\beta^{\prime}(\mu)<0\right)$ ) increasing $\mu$ makes the price more informative about the payoff and decreases (increases) the incentives to acquire information. The result of strategic substitutability $\left(\beta^{\prime}(\mu)>0\right)$ is the one in Grossman and Stiglitz (1980). From the results on comparative statics the following proposition follows.

Proposition 6: If $\rho>0$ the market exhibits strategic substitutability in information acquisition at regular extremal equilibria. When there are three SLE, the market exhibits strategic complementarity in information acquisition in the equilibrium whose value of $\beta$ is intermediate. If $\rho=0$ the market exhibits strategic substitutability (complementarity) in information acquisition at the low (high) $\beta$ regular equilibrium.

The equilibria in which the market exhibits strategic complementarity in information acquisition are unstable. If we discard those, we are left with strategic substitutability in information acquisition as in the Grossman and Stiglitz model. Under adaptive dynamics, starting either at unstable or critical equilibria the system will converge to an equilibrium with larger $\beta$ and $R \cdot{ }^{12}$ In all cases we end up therefore with strategic substitutability in information acquisition.

This result could be modified if agents could acquire information either about the payoff and the noisy supply together or not at all. In this case an equilibrium of the Grossman-Stiglitz type could display strategic complementarity in information acquisition for some configuration of the precision of the signals (see Proposition 5 in Ganguli and Yang (2009) for the case $\rho=0$ ).

\section{$5 \quad$ Particular cases}

The general framework presented above encompasses several of the main models presented in the literature of market microstructure.

Example 1: $\tau_{\eta}=0$ (a generalization of Diamond and Verrecchia (1981)).

This is the classical case of the noise trader model but generalized to allow for correlated errors in the private signals and for the presence of informed and uninformed traders. In this case

$$
f(\beta)=\mu \gamma \frac{\tau_{\varepsilon}^{2}}{\beta^{2} \rho \tau_{u}(1-\rho)+\tau_{\varepsilon}},
$$

$f(\cdot)$ is decreasing in $\beta$ and we have strategic susbtitutability. The intuition of this property is the following. In this example, as in traditional rational expectations models, uninformed traders do not hold any private information, whereas informed traders only have private information about the liquidation

\footnotetext{
${ }^{12}$ An exception is when there is a unique unstable equilibrium (with strong strategic susbtitutability). Then a perturbation would induce divergence.
} 
value of the risky asset. This has two effects. First, uninformed traders' demands depend only on public information, that is, $\Omega^{U}=0$, and hence, $\frac{\partial \Omega^{U}}{\partial \beta}=0$. Second, in relation to informed traders, the precision of private information $\tau^{* I}=\tau_{\varepsilon}$ (and hence, independent of $\beta$ ). This implies that the private learning channel effect of a change in $\beta$ on $\Omega^{I}$ vanishes. Consequently, informed investors' decisions are strategic substitutes in actions, that is, $\frac{\partial \Omega^{I}}{\partial \beta}<0$. Combining these results, as $f(\beta)=\mu \Omega^{I}+(1-\mu) \Omega^{U}$, it follows that $f(\cdot)$ is decreasing in $\beta$.

The fact that the function $f(\cdot)$ is decreasing leads to uniqueness of equilibrium. When there is strong strategic substitutability in actions, the function $f(\cdot)$ is decreasing and it has a very steep slope. In this case the equilibrium is unstable. In the remainder situations the equilibrium is stable. These results are summarized in the following corollary:

Corollary 7: If $\tau_{\eta}=0$, a SLE exists and is unique. The equilibrium is stable if and only if $\frac{4}{\gamma^{2} \mu^{2} \tau_{u} \tau_{\varepsilon}}>(1-\rho) \rho$.

Corollary 7 indicates that when $\rho$ takes extreme values, near 1 or near zero, the equilibrium is stable. This is due to the fact that, when the value of $\rho$ is extreme, the classical public learning channel effect of a change in $\beta$ on $\Omega^{I}$ is moderate. Note that, when $\rho$ is near 0 , the expression $\Omega^{I}=H\left(\tau^{* I}, \tau\right)$ indicates that the effect of $\tau$ on $\Omega^{I}$ is small. On the other hand, when $\rho$ is near 1 , the increase in the public precision, $\tau$, due to a rise in $\beta$ is small. This implies that in both cases the strategic substitutability in actions of informed traders is moderate, and hence, the equilibrium is stable.

Example 2: $\rho=0$ (Ganguli and Yang (2009)).

If the private signal errors are uncorrelated, then

$$
f(\beta)=\mu \gamma\left(\tau_{\eta} \beta^{2}+\tau_{\varepsilon}\right)+(1-\mu) \gamma \beta^{2} \tau_{\eta},
$$

$f(\cdot)$ is increasing in $\beta$ and we have strategic complementarity. In this particular case we can explicitly obtain the equilibrium values for $\beta$.

Corollary 8: Suppose that $\rho=0$. If $1<4 \tau_{\varepsilon} \tau_{\eta} \mu \gamma^{2}$, then a SLE does not exist. Otherwise, that is, if $1 \geq 4 \tau_{\varepsilon} \tau_{\eta} \mu \gamma^{2}$, then we have the following equilibrium values for $\beta$ :

$$
\beta_{1}=\frac{1-\sqrt{1-4 \tau_{\varepsilon} \tau_{\eta} \mu \gamma^{2}}}{2 \tau_{\eta} \gamma} \text { and } \beta_{2}=\frac{1+\sqrt{1-4 \tau_{\varepsilon} \tau_{\eta} \mu \gamma^{2}}}{2 \tau_{\eta} \gamma} .
$$

Moreover, the equilibrium with the lowest value of $\beta$ is stable, whereas the other one is unstable. ${ }^{13}$

\footnotetext{
${ }^{13}$ The stability analysis in the model of Ganguli and Yang (2009) has been performed by Chamley (2008b) and Heinemann (2010). Our result coincides with the one in Chamley (2008b). Note however that the lower equilibrium may be unstable under eductive learning for some parameter configurations (see Heinemann (2010), and Guesnerie (2002) for a general overview of the concept). The lower equilibrium is always stable under adaptive learning and the higher one is not (see Heinemann (2010)).
} 
Corollary 8 shows that when private signals are very precise $\left(\tau_{\varepsilon}\right.$ and $\tau_{\eta}$ high enough) there is no equilibrium. The intuition is the following. If $\tau_{\varepsilon}$ is high enough, although prices are almost not informative ( $\beta$ low), informed investors' weights on private information, $\Omega^{I}$, and, hence, the average weight $f(\beta)$, are high. If this is combined with factors that favor strategic complementarities in actions (for instance, $\tau_{\eta}$ high enough), the result is that there is no intersection between the function $f(\cdot)$ and the 45 -degree line, and hence, there is no equilibrium. Finally, to illustrate the results derived in Corollary 8, we plot the following figure:

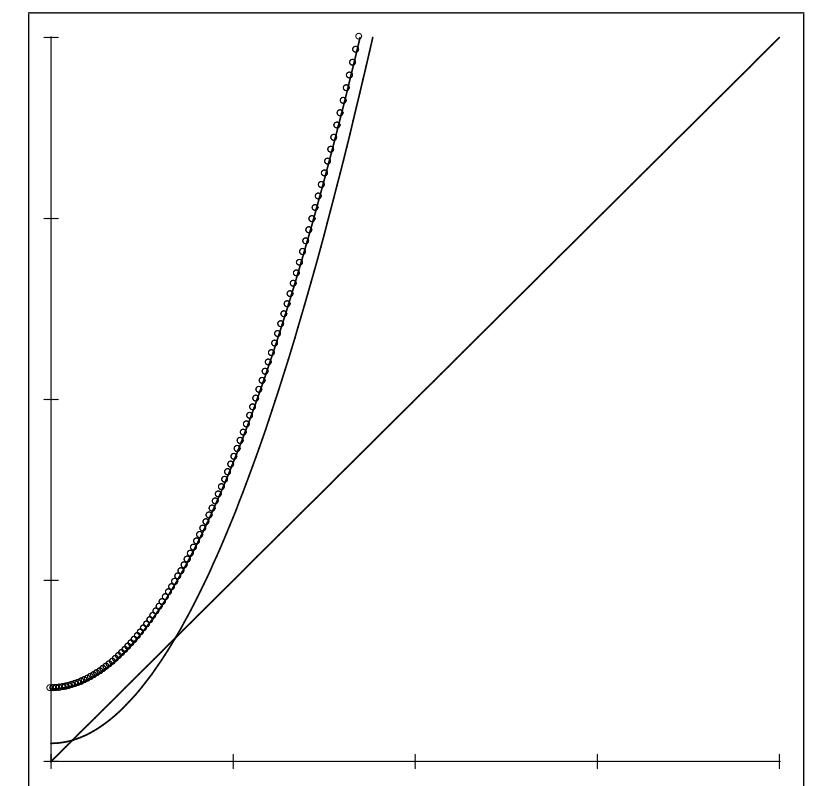

Figure 3. The function $f(\cdot)$ for $\tau_{\varepsilon}=1$ (dotted curve) and $\tau_{\varepsilon}=\frac{1}{4}$ (solid curve), and the 45-degree line (with $\rho=0, \tau_{\eta}=1, \mu=\frac{1}{2}$ and $\gamma=1$ ).

Figure 3 shows that the graph of the function $f(\cdot)$ for $\tau_{\varepsilon}=1$ (dotted curve) is located above the 45-degree line. This implies that when $\tau_{\varepsilon}=1$ there is no equilibrium. By contrast, the graph of the function $f(\cdot)$ for $\tau_{\varepsilon}=\frac{1}{4}$ (solid curve) intersects twice with the 45-degree line, and therefore, in this case there are two equilibria.

Example 3: $\rho=1$ (a generalization of Grossman and Stiglitz (1980)).

In this model (similar to the model in Section 4.4 in Vives (2008)) there are informed (with traders receiving the same signal about $v$ ) and uniformed like in Grossman and Stiglitz (1980) but traders receive another piece of private information on noisy supply. In this case

$$
f(\beta)=\gamma \tau_{\varepsilon}\left(\mu+(1-\mu) \frac{1}{\Psi^{-1}+\tau_{\varepsilon} \tau_{\eta}^{-1} \beta^{-2}}\right)
$$


where $\Psi=\frac{\tau_{\eta}}{\tau_{u}+\tau_{\eta}}, f(\cdot)$ is increasing in $\beta$ and we have strategic complementarity.

Corollary 9: Suppose that $\rho=1$. If $(1-\mu) \Psi=0$, there is a unique SLE. Otherwise,

if $\Psi-\mu(\Psi+8)<0$, there is a unique SLE,

if $\Psi-\mu(\Psi+8)>0$ and $\gamma>\bar{\gamma}$, there is a unique $S L E$,

if $\Psi-\mu(\Psi+8)>0$ and $\gamma<\gamma$, there is a unique SLE,

if $\Psi-\mu(\Psi+8)>0$ and $\gamma=\bar{\gamma}$ or $\gamma=\bar{\gamma}$, there are two SLE,

if $\Psi-\mu(\Psi+8)>0$ and $\gamma \in \overline{(\gamma}, \bar{\gamma})$, there are three $S L E$,

if $\Psi-\mu(\Psi+8)=0$, there is a unique SLE,

where $\underline{\gamma}=\sqrt{\frac{\Psi^{2}(\mu-1)^{2}+\Psi 20 \mu(1-\mu)-8 \mu^{2}-(\Psi-8 \mu-\Psi \mu) \sqrt{\Psi(\mu-1)(8 \mu-\Psi+\Psi \mu)}}{8 \mu\left(\tau_{u}+\tau_{\eta}\right) \tau_{\varepsilon}(\Psi+\mu-\Psi \mu)^{3}}}$ and

$\bar{\gamma}=\sqrt{\frac{\Psi^{2}(\mu-1)^{2}+\Psi 20 \mu(1-\mu)-8 \mu^{2}+(\Psi-8 \mu-\Psi \mu)(\sqrt{\Psi(\mu-1)(8 \mu-\Psi+\Psi \mu)})}{8 \mu\left(\tau_{u}+\tau_{\eta}\right) \tau_{\varepsilon}(\Psi+\mu-\Psi \mu)^{3}}}$.

Before interpreting the results stated in Corollary 9, we plot several figures illustrating the different situations when $\rho=1$.

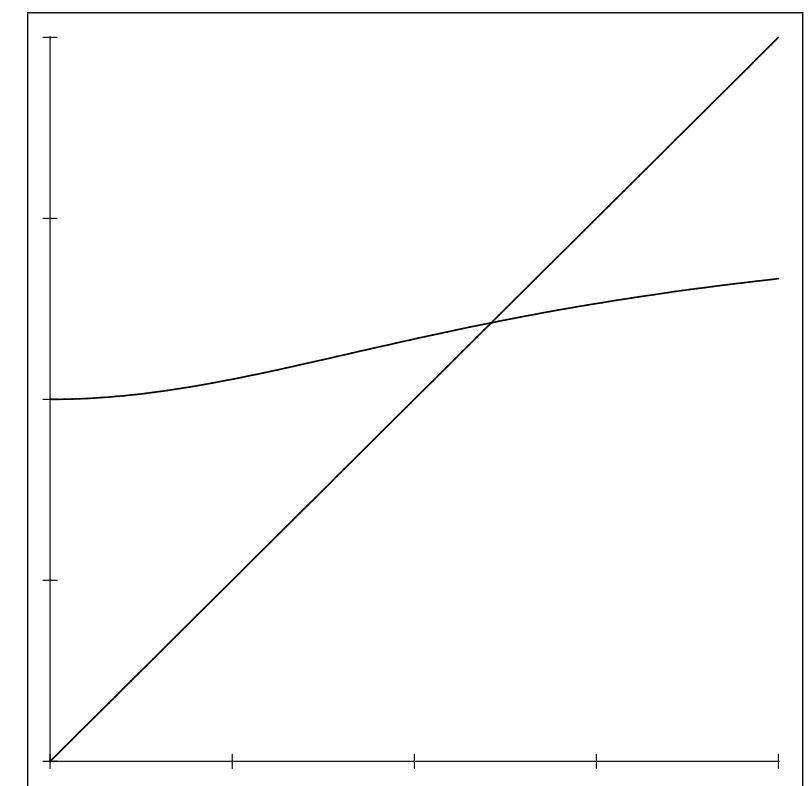

Figure 4. The function $f($.$) and the 45-degree line (with \rho=1, \tau_{\eta}=1$,

$$
\left.\tau_{u}=1, \mu=\frac{1}{2}, \tau_{\varepsilon}=1 \text { and } \gamma=1\right) .
$$

In Figure 4 , the selected parameter values satisfy $\Psi-\mu(\Psi+8)<0$. Consistent with Corollary 9 there is a unique equilibrium.

Figure 2 illustrates a particular case in which $\Psi-\mu(\Psi+8)=0$. As Corollary 9 indicates, in this case that independent of the value of $\gamma$, there is a unique equilibrium. 


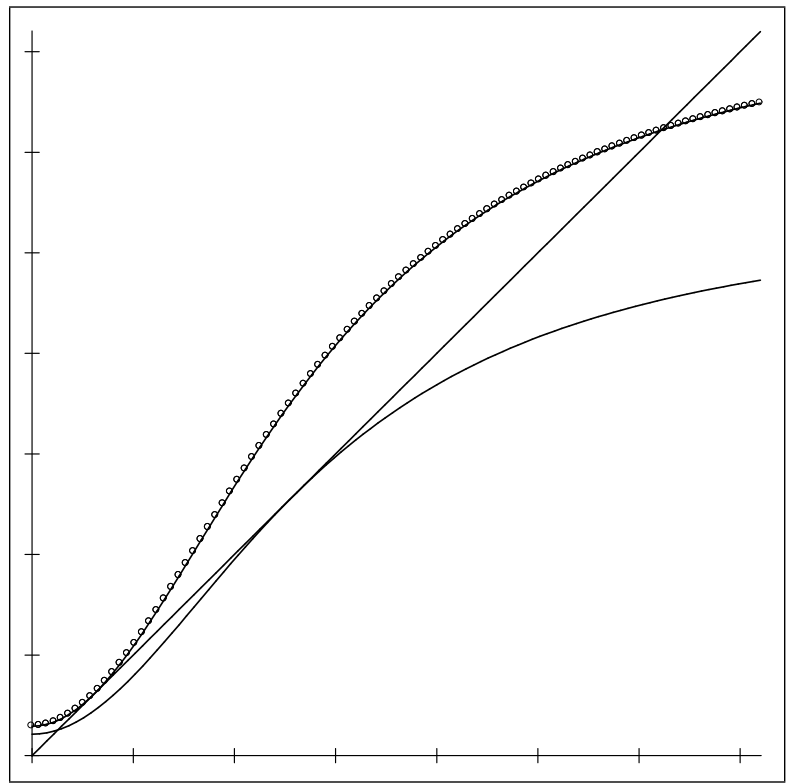

Figure 5. The function $f(\cdot)$ for $\gamma=\bar{\gamma}=3.6503$ (dotted curve) and $\gamma=$ $\underline{\gamma}=2.6594$ (solid curve), where $\rho=1, \tau_{\eta}=1, \tau_{u}=1, \mu=\frac{1}{50}$ and $\tau_{\varepsilon}=1$.

Figure 5 shows that when the parameter $\gamma$ takes the critical values, $\underline{\gamma}$ and $\bar{\gamma}$, there are two equilibria. Figure 1 shows that when $\gamma \notin(\underline{\gamma}, \bar{\gamma})$, there is a unique equilibrium, whereas when $\gamma \in(\underline{\gamma}, \bar{\gamma})$ there are three equilibria.

The intuition of the results is as follows. Notice that if $\rho=1$, the price does not provide any extra information about $v$ to informed traders, and consequently, informed investors only take into account their private signal about $v$. Hence, $\Omega^{I}$ does not vary with $\beta$. If $\mu$ is high enough or $\Psi$ is low enough (because of $\tau_{\eta}$ is low or $\tau_{u}$ is high), on aggregate, strategic complementarities in actions are moderate and this leads to a unique equilibrium. Otherwise, if $\mu$ is low enough or $\Psi$ is high enough, then the number of equilibria will depend on the investors' risk tolerance coefficient $\gamma$. Generically, there are three possibilities:

1) When $\gamma$ is very high $(\gamma>\bar{\gamma})$, strategic complementarities are very strong and the function $f(\cdot)$ has a sharply increasing S-shape. It has a unique intersection with the 45-degree line in the upper branch of the $\mathrm{S}$, and consequently, uniqueness of the equilibrium.

2) For a middle value of $\gamma(\gamma \in(\gamma, \bar{\gamma}))$, the S-shape rises more slowly and we get 3 intersections, and consequently, three equilibria.

3 ) If $\gamma$ is very low $(\gamma<\gamma)$, strategic complementarities in actions are moderate. Hence, the function $\bar{f}(\cdot)$ is increasing, but very flat. Consequently, there is a unique intersection, and hence, a unique equilibrium.

With regard to the stability of equilibria, since $f^{\prime}(\cdot)>0$ we know that regular extremal equilibria are stable and whenever there are three equilibria the middle one is unstable. In case of two $S L E$, there is one stable equilibrium and one unstable equilibrium (with $f^{\prime}(\beta)=1$ ). Note that there can be a single 
equilibrium and be unstable (as in the middle branch in Figure 2).

Example 4: $\mu=1$ (all the investors are informed about the liquidation value).

We have that

$$
f(\beta)=\gamma \tau_{\varepsilon} \frac{\Psi \beta^{2}(1-\rho)+\frac{\tau_{\varepsilon}}{\tau_{u}+\tau_{\eta}}}{\beta^{2} \rho(1-\rho)+\frac{\tau_{\varepsilon}}{\tau_{u}+\tau_{\eta}}},
$$

where $\Psi=\frac{\tau_{\eta}}{\tau_{u}+\tau_{\eta}}$, and $\operatorname{sign}\left(f^{\prime}(\beta)\right)=\operatorname{sign}((\Psi-\rho)(1-\rho))$. For $\rho<1$ we have that $f^{\prime}(\cdot)>0$ if and only if $\Psi>\rho$. That is, if the (squared) correlation coefficient between $u$ and $u_{i}$ is large in relation to the correlation of private signal errors, then the private learning channel from prices dominates for informed investors $\left(\frac{\partial \Omega^{I}}{\partial \beta}>0\right)$.

Corollary 10: Suppose that $\mu=1$. If $(\rho-\Psi)(1-\rho) \geq 0$, then $f^{\prime}(\cdot)<0$ and there is a unique SLE. Otherwise,

if $\Psi-9 \rho<0$, there is a unique SLE,

if $\Psi-9 \rho>0$ and $\gamma>\bar{\gamma}$, there is a unique $S L E$,

if $\Psi-9 \rho>0$ and $\gamma<\underline{\gamma}$, there is a unique $S L E$,

if $\Psi-9 \rho>0$ and $\gamma=\bar{\gamma}$ or $\gamma=\bar{\gamma}$, there are two SLE,

if $\Psi-9 \rho>0$ and $\gamma \in \overline{(\gamma}, \bar{\gamma})$, there are three $S L E$,

if $\Psi-9 \rho=0$, there is a unique $S L E$,

where

$$
\begin{aligned}
& \underline{\gamma}=\sqrt{\frac{\left(18 \rho \Psi-27 \rho^{2}+\Psi^{2}\right)-(\Psi-9 \rho) \sqrt{(\Psi-9 \rho)(\Psi-\rho)}}{8 \Psi(1-\rho) \Psi^{2} \tau_{\varepsilon}\left(\tau_{u}+\tau_{\eta}\right)}} \text { and } \\
& \bar{\gamma}=\sqrt{\frac{\left(18 \rho \Psi-27 \rho^{2}+\Psi^{2}\right)+(\Psi-9 \rho) \sqrt{(\Psi-9 \rho)(\Psi-\rho)}}{8 \Psi(1-\rho) \Psi^{2} \tau_{\varepsilon}\left(\tau_{u}+\tau_{\eta}\right)}}
\end{aligned}
$$

Again, in order to illustrate results stated in Corollary 10, we plot several figures. 


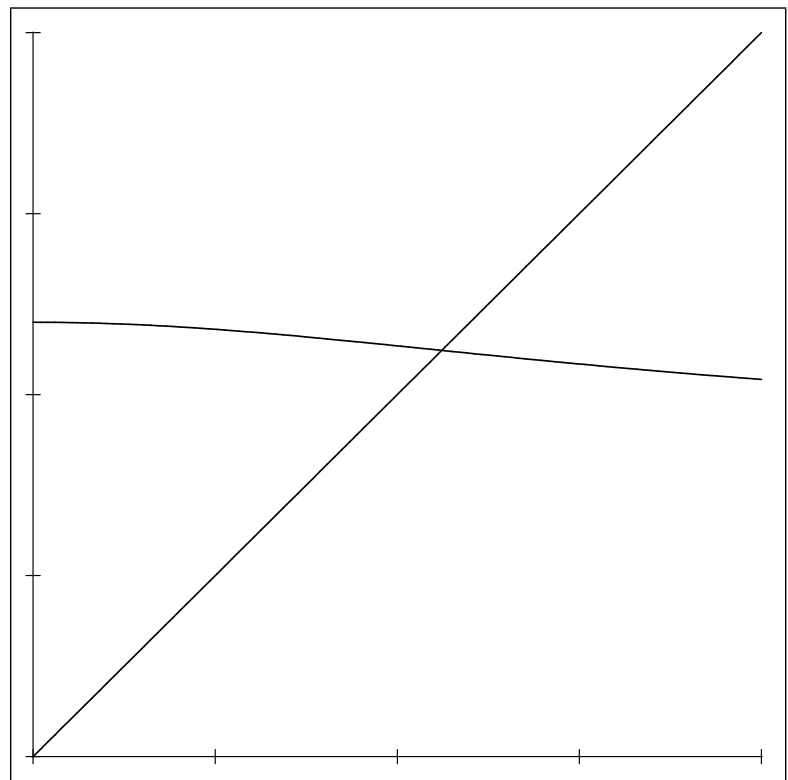

Figure 6. The function $f(\cdot)$ and the 45 -line where $\mu=1, \rho=\frac{2}{3}, \tau_{\eta}=1$,

$$
\tau_{u}=1, \tau_{\varepsilon}=\frac{2}{5} \text { and } \gamma=\frac{3}{2} .
$$

The parameters values used in Figure 6 satisfy $(\rho-\Psi)(1-\rho) \geq 0$ and consistently with Corollary 10, investors' decisions are strategic substitutes, $f(\cdot)$ is decreasing, and there is a unique equilibrium.

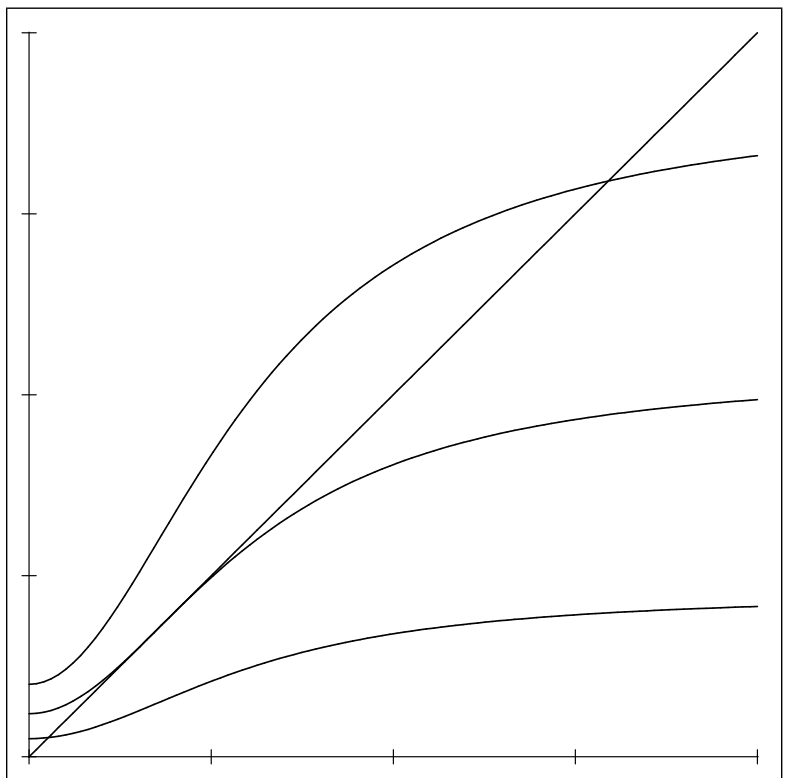

Figure 7. The function $f(\cdot)$ for $\gamma=\frac{1}{4}, \gamma=\underline{\gamma}=\bar{\gamma}=0.59409$, and $\gamma=1$, where $\mu=1, \rho=\frac{1}{18}, \tau_{\eta}=1, \tau_{u}=1$ and $\tau_{\varepsilon}=1$. 
In Figure 7, the selected parameter values satisfy $\Psi-9 \rho=0$. As Corollary 10 indicates, whenever $\Psi-9 \rho=0$, independent of the value of $\gamma$, there is a unique equilibrium.

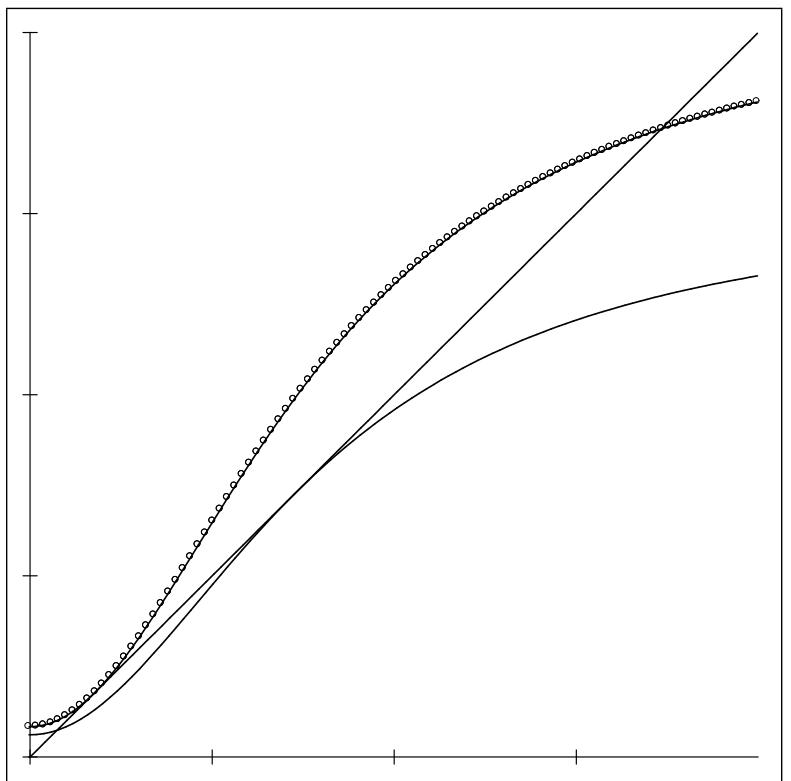

Figure 8. The function $f(\cdot)$ when $\gamma=\gamma=0.61215$ (solid curve) and when $\gamma=\bar{\gamma}=0.83345$ (dotted curve), where $\mu=1, \rho=\frac{1}{50}, \tau_{\eta}=1, \tau_{u}=1$ and

$$
\tau_{\varepsilon}=\frac{2}{5} \text {. }
$$

In Figure 8, the parameter $\gamma$ takes the critical values, $\gamma$ and $\bar{\gamma}$. In these cases there are two equilibria. For all $\gamma>\bar{\gamma}(\gamma<\underline{\gamma})$, the graph of $f(\cdot)$ is located above (below) the dotted (solid) curve, and we get a unique intersection with the 45degree line. For all $\gamma \in(\underline{\gamma}, \bar{\gamma})$, the graph of $f(\cdot)$ is located between the solid and the dotted curve and we will have three intersections with the 45-degree line. This is illustrated in Figure 9, assuming that $\gamma=0.5, \gamma=0.7$ and $\gamma=1$. 


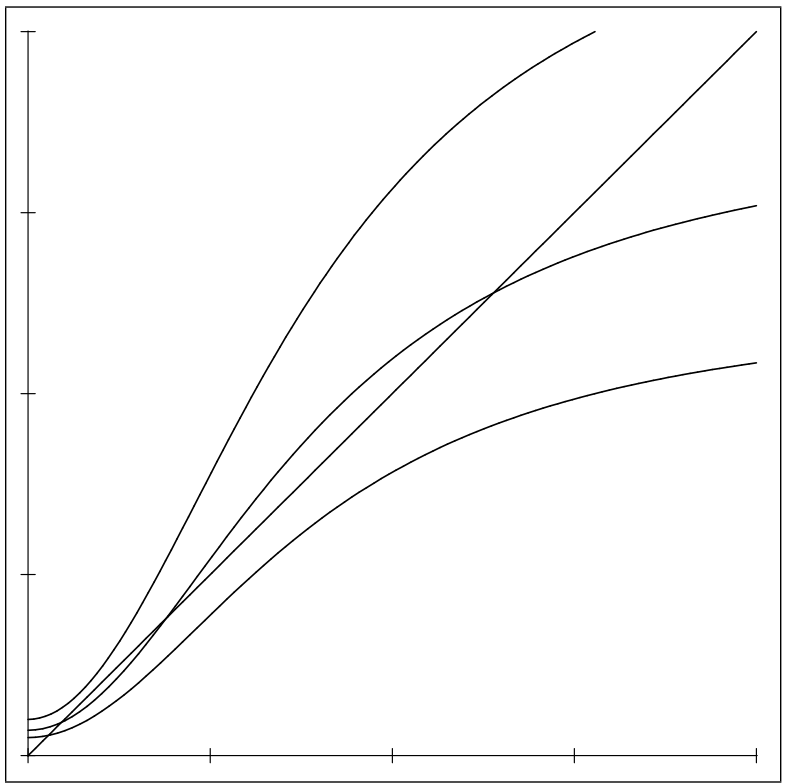

Figure 9. The function $f(\cdot)$ for $\gamma=0.5, \gamma=0.7$, and $\gamma=1$, where $\mu=1$,

$$
\rho=\frac{1}{50}, \tau_{\eta}=1, \tau_{u}=1 \text { and } \tau_{\varepsilon}=\frac{2}{5} .
$$

From the last two figures, we obtain that:

1) When $\gamma$ is very high $(\gamma>\bar{\gamma})$, strategic complementarities in actions are very strong and the function $f(\cdot)$ has a sharply increasing S-shape. It has a unique intersection with the 45-degree line in the upper branch of the $\mathrm{S}$, and consequently, uniqueness of the equilibrium.

2) For a middle value of $\gamma(\gamma \in(\underline{\gamma}, \bar{\gamma}))$, the S-shape rises more slowly and we get 3 intersections, and consequently, three equilibria.

1) If $\gamma$ is very low $(\gamma<\gamma)$, strategic complementarities in actions are moderate. Hence, the function $\bar{f}(\cdot)$ is increasing but very flat. Consequently, it has a unique intersection, and hence, uniqueness of the equilibrium.

Let us examine the stability of equilibria.

Corollary 11: Suppose that $\mu=1,0<\rho<1$ and $\Psi>0$.

a) If $\rho \leq \Psi$, we have strategic complementarity, regular extremal equilibria are stable and whenever there are three equilibria the middle one is unstable. In case of two SLE, there is one stable equilibrium and one unstable equilibrium (with $f^{\prime}(\beta)=1$ ).

b) If $\Psi<\rho$, we have strategic susbtitutability and

b.1) if $\Psi<\rho \leq 9 \Psi$, the unique equilibrium is stable.

b.2) if $\rho>9 \Psi$, then we have two possibilities

if $\gamma \notin\left(\gamma_{1}, \gamma_{2}\right)$, the equilibrium is stable, 
if $\gamma \in\left[\gamma_{1}, \gamma_{2}\right]$, the equilibrium is unstable, where

$$
\begin{aligned}
& \gamma_{1}=\sqrt{\frac{\rho\left(12 \Psi^{2}-9 \Psi \rho+\rho^{2}+(4 \Psi-\rho)(\sqrt{(\rho-9 \Psi)(\rho-\Psi)})\right.}{\Psi^{3}(1-\rho)(-9 \Psi+5 \rho-3 \sqrt{(\rho-9 \Psi)(\rho-\Psi)}) \tau_{\varepsilon}\left(\tau_{u}+\tau_{\eta}\right)}} \text { and } \\
& \gamma_{2}=\sqrt{\frac{\rho\left(12 \Psi^{2}-9 \Psi \rho+\rho^{2}+(\rho-4 \Psi) \sqrt{(\rho-9 \Psi)(\rho-\Psi)}\right.}{(1-\rho) \Psi^{3}(-9 \Psi+5 \rho+3 \sqrt{(\rho-9 \Psi)(\rho-\Psi)}) \tau_{\varepsilon}\left(\tau_{u}+\tau_{\eta}\right)}}
\end{aligned}
$$

The results in this section confirm the forces (parameter constellations) for strategic substitutability, and uniqueness of equilibrium therefore. Those are when the fraction of informed traders is large ( $\mu$ high) and the correlation among the private signal errors is high ( $\rho$ high), or when investors almost do not hold information about the aggregate supply ( $\Psi$ low $)$. The same forces together when informed investors have a low risk weighted information advantage about $v\left(\gamma \tau_{\varepsilon}\right.$ low) push for low strategic complementarity. To those we add the case where informed investors have a very precise information about $v\left(\tau_{\varepsilon}\right.$ very high).

\section{Concluding remarks}

This paper suggests that the type of information observed by market participants affects the existence and the number of partially revealing rational expectations equilibria. Strong strategic complementarities in the actions of investors are the driver of multiplicity of equilibria. A necessary condition for multiplicity of equilibria is the presence of multiple sources of asymmetric information, leading to a private learning channel from public signals. This channel induces strategic complementarities in the actions of investors and when it is strong enough it overpowers the classical effect of more public information implying less weight on private signals, leading to strategic substitutability in actions.

Several extensions are left for future research. A first one is to develop the welfare analysis in our market. A second one is to explore the dynamic implications of the model. A third one is to develop the analysis in a framework with strategic traders instead of price-taking ones.

\section{Appendix}

Proof of Proposition 1: Consider the informed trader $i$. Suppose that this agent conjectures the functional form of the price given by (1). The maximization problem of this investor is

$$
\max _{x_{i}^{I}} E\left(-e^{-\frac{1}{\gamma} W_{i}^{I}} \mid s_{i}, u_{i}, p\right),
$$


which is equivalent to

$$
\max _{x_{i}^{I}} E\left(W_{i}^{I} \mid s_{i}, u_{i}, p\right)-\frac{1}{2 \gamma} \operatorname{var}\left(W_{i}^{I} \mid s_{i}, u_{i}, p\right) .
$$

Using the expression of the final wealth of this agent, we have

$$
\begin{aligned}
E\left(W_{i}^{I} \mid s_{i}, u_{i}, p\right) & =E\left(v \mid s_{i}, u_{i}, p\right) u_{i}+\left(E\left(v \mid s_{i}, u_{i}, p\right)-p\right) x_{i}^{I} \text { and } \\
\operatorname{var}\left(W_{i}^{I} \mid s_{i}, u_{i}, p\right) & =\left(x_{i}^{I}+u_{i}\right)^{2} \operatorname{var}\left(v \mid s_{i}, u_{i}, p\right) .
\end{aligned}
$$

Substituting these expressions in the objective function and maximizing with respect to $x_{i}^{I}$, the first order condition implies that

$$
x_{i}^{I}=\gamma \frac{E\left(v \mid s_{i}, u_{i}, p\right)-p-\frac{1}{\gamma} \operatorname{var}\left(v \mid s_{i}, u_{i}, p\right) u_{i}}{\operatorname{var}\left(v \mid s_{i}, u_{i}, p\right)} .
$$

Applying standard normal theory, we have

$$
\begin{aligned}
& E\left(v \mid s_{i}, u_{i}, p\right)= \\
& =\bar{v}+\frac{\left.\tau_{\varepsilon}^{2} A_{2}^{2}\left(s_{i}-\bar{v}\right)+(1-\rho) \tau_{\varepsilon} \tau_{\eta} A_{1} A_{2}\left(u_{i}-\bar{u}\right)+(1-\rho)\left(\tau_{u}+\tau_{\eta}\right) \tau_{\varepsilon} A_{1}\left(p-\left(A_{0}+A_{1} \bar{v}-A_{2} \bar{u}\right)\right)\right)}{\left(\tau_{\varepsilon}+\rho \tau_{v}\right)(1-\rho)\left(\tau_{u}+\tau_{\eta}\right) A_{1}^{2}+\tau_{\varepsilon}\left(\tau_{v}+\tau_{\varepsilon}\right) A_{2}^{2}} \text { and } \\
& \left(\tau^{I}\right)^{-1}=\operatorname{var}\left(v \mid s_{i}, u_{i}, p\right)=\frac{\tau_{\varepsilon} A_{2}^{2}+\rho(1-\rho)\left(\tau_{u}+\tau_{\eta}\right) A_{1}^{2}}{\left(\tau_{\varepsilon}+\rho \tau_{v}\right)(1-\rho)\left(\tau_{u}+\tau_{\eta}\right) A_{1}^{2}+\tau_{\varepsilon}\left(\tau_{v}+\tau_{\varepsilon}\right) A_{2}^{2}}
\end{aligned}
$$

Substituting these expressions into (9), we get

$$
x_{i}^{I}=B_{0}-B_{1} u_{i}-B_{2} p+B_{3} s_{i},
$$

with

$$
\begin{aligned}
B_{0} & =\gamma\left(\tau_{v} \bar{v}+\frac{(1-\rho) \tau_{\varepsilon} A_{1}\left(-\left(\tau_{u}+\tau_{\eta}\right) A_{0}+\tau_{u} A_{2} \bar{u}\right)}{\rho(1-\rho)\left(\tau_{u}+\tau_{\eta}\right) A_{1}^{2}+\tau_{\varepsilon} A_{2}^{2}}\right) \\
B_{1} & =1-\frac{\gamma(1-\rho) \tau_{\varepsilon} \tau_{\eta} A_{1} A_{2}}{\rho(1-\rho)\left(\tau_{u}+\tau_{\eta}\right) A_{1}^{2}+\tau_{\varepsilon} A_{2}^{2}}, \\
B_{2} & =\gamma \frac{(1-\rho)\left(\tau_{u}+\tau_{\eta}\right)\left(A_{1}\left(\tau_{\varepsilon}+\rho \tau_{v}\right)-\tau_{\varepsilon}\right) A_{1}+A_{2}^{2} \tau_{\varepsilon}\left(\tau_{v}+\tau_{\varepsilon}\right)}{\rho(1-\rho)\left(\tau_{u}+\tau_{\eta}\right) A_{1}^{2}+\tau_{\varepsilon} A_{2}^{2}} \text { and } \\
B_{3} & =\gamma \frac{\tau_{\varepsilon}^{2} A_{2}^{2}}{\rho(1-\rho)\left(\tau_{u}+\tau_{\eta}\right) A_{1}^{2}+\tau_{\varepsilon} A_{2}^{2}} .
\end{aligned}
$$

Consider now the uninformed trader $j$. Suppose that this agent conjectures that the price has the functional form given in (1). The maximization problem of this investor is

$$
\max _{x_{j}^{U}} E\left(-e^{-\frac{1}{\gamma} W_{j}^{U}} \mid u_{j}, p\right),
$$

which is equivalent to the following maximization problem:

$$
\max _{x_{j}^{U}} E\left(W_{j}^{U} \mid u_{j}, p\right)-\frac{1}{2 \gamma} \operatorname{var}\left(W_{j}^{U} \mid u_{j}, p\right) .
$$


Using the expression of the final wealth of this agent, we have

$$
\begin{aligned}
E\left(W_{j}^{U} \mid u_{j}, p\right) & =E\left(v \mid u_{j}, p\right) u_{j}+\left(E\left(v \mid u_{j}, p\right)-p\right) x_{j}^{U} \text { and } \\
\operatorname{var}\left(W_{j}^{U} \mid u_{j}, p\right) & =\left(x_{j}^{U}+u_{j}\right)^{2} \operatorname{var}\left(v \mid u_{j}, p\right) .
\end{aligned}
$$

Substituting these expressions in the objective function and maximizing with respect to $x_{j}^{U}$, the first order condition implies that

$$
x_{j}^{U}=\gamma \frac{E\left(v \mid u_{j}, p\right)-p-\frac{1}{\gamma} \operatorname{var}\left(v \mid u_{j}, p\right) u_{j}}{\operatorname{var}\left(v \mid u_{j}, p\right)} .
$$

Again applying standard normal theory, we get

$$
\begin{aligned}
& E\left(v \mid u_{j}, p\right)=\bar{v}+\frac{\tau_{\varepsilon} \tau_{\eta} A_{2} A_{1}\left(u_{j}-\bar{u}\right)+\left(\tau_{u}+\tau_{\eta}\right) \tau_{\varepsilon} A_{1}\left(p-\left(A_{0}+A_{1} \bar{v}-A_{2} \bar{u}\right)\right)}{\left(\tau_{\varepsilon}+\rho \tau_{v}\right)\left(\tau_{u}+\tau_{\eta}\right) A_{1}^{2}+\tau_{v} \tau_{\varepsilon} A_{2}^{2}}, \\
& \left(\tau^{U}\right)^{-1}=\operatorname{var}\left(v \mid u_{j}, p\right)=\frac{\left(A_{2}^{2} \tau_{\varepsilon}+A_{1}^{2} \rho\left(\tau_{u}+\tau_{\eta}\right)\right)}{\left(\tau_{\varepsilon}+\rho \tau_{v}\right)\left(\tau_{u}+\tau_{\eta}\right) A_{1}^{2}+\tau_{v} \tau_{\varepsilon} A_{2}^{2}} .
\end{aligned}
$$

Plugging these expressions into (10), we have

$$
x_{j}^{U}=C_{0}-C_{1} u_{j}-C_{2} p,
$$

with

$$
\begin{aligned}
C_{0} & =\gamma\left(\tau_{v} \bar{v}+\frac{\tau_{u} \tau_{\varepsilon} \bar{u} A_{1} A_{2}-\tau_{\varepsilon}\left(\tau_{u}+\tau_{\eta}\right) A_{1} A_{0}}{\rho\left(\tau_{u}+\tau_{\eta}\right) A_{1}^{2}+\tau_{\varepsilon} A_{2}^{2}}\right), \\
C_{1} & =1-\frac{\gamma \tau_{\eta} \tau_{\varepsilon} A_{1} A_{2}}{\rho\left(\tau_{u}+\tau_{\eta}\right) A_{1}^{2}+\tau_{\varepsilon} A_{2}^{2}} \text { and } \\
C_{2} & =\gamma \frac{\left(\tau_{u}+\tau_{\eta}\right)\left(\left(\tau_{\varepsilon}+\rho \tau_{v}\right) A_{1}-\tau_{\varepsilon}\right) A_{1}+\tau_{v} \tau_{\varepsilon} A_{2}^{2}}{\rho\left(\tau_{u}+\tau_{\eta}\right) A_{1}^{2}+\tau_{\varepsilon} A_{2}^{2}} .
\end{aligned}
$$

Using the optimal demands for all investors, the market clearing condition implies that

$$
p=\frac{\mu B_{0}+(1-\mu) C_{0}-\left(\mu B_{1}+(1-\mu) C_{1}\right) u+\mu B_{3}(v+\widetilde{\varepsilon})}{\mu B_{2}+(1-\mu) C_{2}} .
$$

Equating coefficients according to (1)

$$
\begin{aligned}
& A_{0}=\frac{\mu B_{0}+(1-\mu) C_{0}}{\mu B_{2}+(1-\mu) C_{2}}, \\
& A_{1}=\frac{\mu B_{3}}{\mu B_{2}+(1-\mu) C_{2}} \text { and } \\
& A_{2}=\frac{\mu B_{1}+(1-\mu) C_{1}}{\mu B_{2}+(1-\mu) C_{2}} .
\end{aligned}
$$

Using the expressions of the coefficients $B$ 's and $C$ 's, we have

$$
\frac{A_{1}}{A_{2}}=\frac{\mu \gamma \frac{\tau_{\varepsilon}^{2} A_{2}^{2}}{\rho(1-\rho)\left(\tau_{u}+\tau_{\eta}\right) A_{1}^{2}+\tau_{\varepsilon} A_{2}^{2}}}{1-\tau_{\varepsilon} \tau_{\eta} \gamma\left(\mu \frac{(1-\rho)}{\rho(1-\rho)\left(\tau_{u}+\tau_{\eta}\right) A_{1}^{2}+\tau_{\varepsilon} A_{2}^{2}}+(1-\mu) \frac{1}{\rho\left(\tau_{u}+\tau_{\eta}\right) A_{1}^{2}+\tau_{\varepsilon} A_{2}^{2}}\right) A_{1} A_{2}} .
$$


Let $\beta=\frac{A_{1}}{A_{2}}$. Operating the previous equality, we have that $\beta$ is a root of the following polynomial

$$
P(\beta)=c_{5} \beta^{5}+c_{4} \beta^{4}+c_{3} \beta^{3}+c_{2} \beta^{2}+c_{1} \beta+c_{0},
$$

where

$$
\begin{aligned}
c_{5} & =\rho^{2}(1-\rho)\left(\tau_{u}+\tau_{\eta}\right)^{2}, \\
c_{4} & =\rho(\rho-1) \tau_{\varepsilon} \tau_{\eta}\left(\tau_{u}+\tau_{\eta}\right) \gamma, \\
c_{3} & =\rho(2-\rho)\left(\tau_{u}+\tau_{\eta}\right) \tau_{\varepsilon}, \\
c_{2} & =-\tau_{\varepsilon}^{2} \gamma\left(\tau_{\eta}+\rho \mu \tau_{u}\right), \\
c_{1} & =\tau_{\varepsilon}^{2}, \text { and } \\
c_{0} & =-\mu \gamma \tau_{\varepsilon}^{3} .
\end{aligned}
$$

Straightforward computations yield that $\beta$ is a zero of the polynomial $P(\beta)$ iff $\beta$ is a solution of the fixed-point equation stated in this proposition. The result related to the existence of a $S L E$ is trivial. It follows from the fact that $f(0)=\gamma \mu \tau_{\varepsilon}>0$ and $\lim _{\beta \rightarrow \infty} f(\beta)<\infty$ whenever $\rho \neq 0$

Next, we derive the expressions of $A_{2}, A_{1}$ and $A_{0}$ as functions of $\beta$. Using the expressions of the coefficients $B$ 's and $C$ 's, from (13), direct computations yield the desired expression for $A_{2}$.

Concerning the expression of $A_{1}$, notice that substituting the expression of $\tau^{I}$ and $\tau^{U}$ in $1-\frac{\tau_{v}}{\left(\mu \tau^{I}+(1-\mu) \tau^{U}\right)}$ and operating, we get

$$
1-\frac{\tau_{v}}{\mu \tau^{I}+(1-\mu) \tau^{U}}=\frac{\mu \frac{\left(A_{1}^{2}\left(\tau_{u}+\tau_{\eta}\right)(1-\rho)+\tau_{\varepsilon} A_{2}^{2}\right) \tau_{\varepsilon}}{\rho(1-\rho)\left(\tau_{u}+\tau_{\eta}\right) A_{1}^{2}+\tau_{\varepsilon} A_{2}^{2}}+(1-\mu) \frac{\left(\tau_{u}+\tau_{\eta}\right) A_{1}^{2} \tau_{\varepsilon}}{\rho\left(\tau_{u}+\tau_{\eta}\right) A_{1}^{2}+\tau_{\varepsilon} A_{2}^{2}}}{\tau_{v}+\mu \frac{\left(A_{1}^{2}\left(\tau_{u}+\tau_{\eta}\right)(1-\rho)+\tau_{\varepsilon} A_{2}^{2}\right) \tau_{\varepsilon}}{\rho(1-\rho)\left(\tau_{u}+\tau_{\eta}\right) A_{1}^{2}+\tau_{\varepsilon} A_{2}^{2}}+(1-\mu) \frac{\left(\tau_{u}+\tau_{\eta}\right) A_{1}^{2} \tau_{\varepsilon}}{\rho\left(\tau_{u}+\tau_{\eta}\right) A_{1}^{2}+\tau_{\varepsilon} A_{2}^{2}}} .
$$

Using (12),

$$
\begin{aligned}
& \mu \frac{\tau_{\varepsilon}^{2} A_{2}^{2}}{\rho(1-\rho)\left(\tau_{u}+\tau_{\eta}\right) A_{1}^{2}+\tau_{\varepsilon} A_{2}^{2}}= \\
& A_{1}\left(\frac{\mu\left((1-\rho)\left(\tau_{u}+\tau_{\eta}\right)\left(\tau_{\varepsilon}\left(A_{1}-1\right)+\rho \tau_{v} A_{1}\right) A_{1}+A_{2}^{2} \tau_{\varepsilon}\left(\tau_{v}+\tau_{\varepsilon}\right)\right)}{\rho(1-\rho)\left(\tau_{u}+\tau_{\eta}\right) A_{1}^{2}+\tau_{\varepsilon} A_{2}^{2}}+\frac{(1-\mu)\left(\left(\tau_{u}+\tau_{\eta}\right)\left(\left(\tau_{\varepsilon}+\rho \tau_{v}\right) A_{1}-\tau_{\varepsilon}\right) A_{1}+\tau_{v} \tau_{\varepsilon} A_{2}^{2}\right)}{\rho\left(\tau_{u}+\tau_{\eta}\right) A_{1}^{2}+\tau_{\varepsilon} A_{2}^{2}}\right) .
\end{aligned}
$$

Substituting this expression in the RHS of the previous equality and operating, we get $1-\frac{\tau_{v}}{\mu \tau^{I}+(1-\mu) \tau^{U}}=A_{1}$.

In relation to the expression of $A_{0}$, note that isolating $A_{0}$ from (11), operating taking into account the expressions of $A_{1}$ and $A_{2}$, we get the desired formula for $A_{0}$.

Finally, concerning the upper bound for the number of $S L E$, we have that the number of $S L E$ is the same as the number of the roots of the polynomial $P(\beta)$. Notice that if $\rho$ is either 0 or 1 , then $P(\beta)$ has degree two or three. Therefore, the result is trivial. Suppose that $\rho \neq 0,1$. By virtue of Rolle's Theorem, to show the result it is enough to prove that there exists a unique $\beta$ such that $P^{\prime \prime}(\beta)=0$. Differentiating we have

$$
\begin{aligned}
P^{\prime \prime}(\beta) & =20 c_{5} \beta^{3}+12 c_{4} \beta^{2}+6 c_{3} \beta+2 c_{2} \text { and } \\
P^{\prime \prime \prime}(\beta) & =60 c_{5} \beta^{2}+24 c_{4} \beta+6 c_{3} .
\end{aligned}
$$


We distinguish three cases:

Case 1: $2 \gamma^{2}(1-\rho) \tau_{\varepsilon} \tau_{\eta}^{2}<5(2-\rho) \rho\left(\tau_{u}+\tau_{\eta}\right)$.

In this case $P^{\prime \prime \prime}(\beta)$ hasn't real roots. The fact that $c_{3}>0$ implies that $P^{\prime \prime \prime}(\beta)>0$ for all $\beta$, and consequently, $P^{\prime \prime}(\beta)$ is strictly increasing. Moreover, since $\lim _{\beta \rightarrow-\infty} P^{\prime \prime}(\beta)=-\infty$ and $\lim _{\beta \rightarrow+\infty} P^{\prime \prime}(\beta)=+\infty$, we conclude that in this case $P^{\prime \prime}(\beta)$ has a unique real root.

Case 2: $2 \gamma^{2}(1-\rho) \tau_{\varepsilon} \tau_{\eta}^{2}=5(2-\rho) \rho\left(\tau_{u}+\tau_{\eta}\right)$.

In this case $P^{\prime \prime \prime}(\beta)$ has a unique real root, denoted by $\beta^{\prime}$. The fact that $c_{3}>0$ implies that $P^{\prime \prime \prime}(\beta)>0$ for all $\beta \neq \beta^{\prime}$, and consequently, $P^{\prime \prime}(\beta)$ is strictly increasing for all $\beta \neq \beta^{\prime}$. As before, since $\lim _{\beta \rightarrow-\infty} P^{\prime \prime}(\beta)=-\infty$ and $\lim _{\beta \rightarrow+\infty} P^{\prime \prime}(\beta)=+\infty$, we conclude that in this case $P^{\prime \prime}(\beta)$ has a unique real root.

Case 3: $2 \gamma^{2}(1-\rho) \tau_{\varepsilon} \tau_{\eta}^{2}>5(2-\rho) \rho\left(\tau_{u}+\tau_{\eta}\right)$.

In this case $P^{\prime \prime \prime}(\beta)$ has two real roots, given by:

$\beta_{1}=\frac{2 \tau_{\varepsilon} \tau_{\eta}(1-\rho) \gamma-\sqrt{2} \sqrt{(1-\rho) \tau_{\varepsilon}\left(\tau_{\varepsilon} 2 \tau_{\eta}^{2} \gamma^{2}(1-\rho)-5 \rho\left(\tau_{u}+\tau_{\eta}\right)(2-\rho)\right)}}{10(1-\rho) \rho\left(\tau_{u}+\tau_{\eta}\right)}$

and

$\beta_{2}=\frac{2 \tau_{\varepsilon} \tau_{\eta}(1-\rho) \gamma+\sqrt{2} \sqrt{(1-\rho) \tau_{\varepsilon}\left(\tau_{\varepsilon} 2 \tau_{\eta}^{2} \gamma^{2}(1-\rho)-5 \rho\left(\tau_{u}+\tau_{\eta}\right)(2-\rho)\right)}}{10(1-\rho) \rho\left(\tau_{u}+\tau_{\eta}\right)}$,

where $\beta_{1}$ is a local maximum of $P^{\prime \prime}(\beta)$. Using the fact that $\tau_{\varepsilon}>\frac{5(2-\rho)\left(\tau_{u}+\tau_{\eta}\right) \rho}{2 \gamma^{2}(1-\rho) \tau_{\eta}^{2}}$, and after some tedious computations, we obtain that $P^{\prime \prime}\left(\beta_{1}\right)<0$. Hence, we have that $P^{\prime \prime}(\beta)$ has a unique root.

Proof of Proposition 3: a) In order to prove uniqueness, it suffices to show that the inequality given in the statement of the corollary guarantees that the function $f(\beta)$ is decreasing for all $\beta \geq 0$. Doing some computations we have that $f^{\prime}(\beta) \leq 0$ is equivalent to $\left(\frac{\beta^{2} \rho\left(\tau_{u}+\tau_{\eta}\right)(1-\rho)+\tau_{\varepsilon}}{\beta^{2} \rho\left(\tau_{u}+\tau_{\eta}\right)+\tau_{\varepsilon}}\right)^{2} \leq \mu \frac{\left(\rho \tau_{u}-\tau_{\eta}+\rho \tau_{\eta}\right)(1-\rho)}{(1-\mu) \tau_{\eta}}$, or using the expression of $\Psi,\left(\frac{\beta^{2} \rho\left(\tau_{u}+\tau_{\eta}\right)(1-\rho)+\tau_{\varepsilon}}{\beta^{2} \rho\left(\tau_{u}+\tau_{\eta}\right)+\tau_{\varepsilon}}\right)^{2} \leq \mu \frac{\left(\rho \Psi^{-1}-1\right)(1-\rho)}{(1-\mu)}$. The expression in the LHS of this inequality is a decreasing function in $\beta$, whenever $\beta \geq 0$. Hence, we get that $\left(\frac{\beta^{2} \rho\left(\tau_{u}+\tau_{\eta}\right)(1-\rho)+\tau_{\varepsilon}}{\beta^{2} \rho\left(\tau_{u}+\tau_{\eta}\right)+\tau_{\varepsilon}}\right)^{2} \leq 1$. Thus, if the inequality $1 \leq \mu \frac{\left(\rho \Psi^{-1}-1\right)(1-\rho)}{(1-\mu)}$ holds, we can assure that $f(\beta)$ is decreasing for all $\beta \geq 0$.

b) Recall that $\frac{\partial \Omega^{U}}{\partial \beta}=\gamma \frac{2 \tau_{\eta} \tau_{\varepsilon}^{2} \beta}{\left(\tau_{\varepsilon}+\beta^{2} \rho\left(\tau_{u}+\tau_{\eta}\right)\right)^{2}}$. Differentiating,

$$
\frac{\partial}{\partial \beta}\left(\frac{\partial \Omega^{U}}{\partial \beta}\right)=2 \frac{\left(\tau_{\varepsilon}-3 \rho \beta^{2}\left(\tau_{u}+\tau_{\eta}\right)\right) \gamma \tau_{\varepsilon}^{2} \tau_{\eta}}{\left(\tau_{\varepsilon}+\beta^{2} \rho\left(\tau_{u}+\tau_{\eta}\right)\right)^{3}} .
$$

Hence, $\beta=\sqrt{\frac{\tau_{\varepsilon}}{3 \rho\left(\tau_{u}+\tau_{\eta}\right)}}$ is a maximum. At this point $\frac{\partial \Omega^{U}}{\partial \beta}=\frac{9}{8}\left(\sqrt{\frac{1}{3 \rho} \frac{\tau_{\varepsilon}}{\tau_{u}+\tau_{\eta}}}\right) \tau_{\eta} \gamma$. Therefore, if $\frac{9}{8}\left(\sqrt{\frac{1}{3 \rho} \frac{\tau_{\varepsilon}}{\tau_{u}+\tau_{\eta}}}\right) \tau_{\eta} \gamma \leq 1$, or equivalently, $\frac{27}{64} \frac{\tau_{\eta}^{2} \tau_{\varepsilon} \gamma^{2}}{\left(\tau_{u}+\tau_{\eta}\right) \rho}<1$, then 
$\frac{\partial \Omega^{U}}{\partial \beta}<1$. As $\frac{\partial \Omega^{I}}{\partial \beta} \leq \frac{\partial \Omega^{U}}{\partial \beta}$ always holds, we get that $f^{\prime}(\beta)=\mu \frac{\partial \Omega^{I}}{\partial \beta}+(1-\mu) \frac{\partial \Omega^{U}}{\partial \beta}<$ 1 for all $\beta$, which warrants uniqueness of equilibrium.

Next, we state a lemma that will be applied in the proof of Corollary 5 .

Lemma A.1. Let $z \sim N(0, \Sigma)$ and $W=c+b / z+z \prime A z$, where $c \in R, b \in R^{n}$, and $A$ is an $\mathrm{n} \times \mathrm{n}$ matrix. Then, if $\Sigma^{-1}+2 \rho A$ is positive definite, then

$$
E\left(e^{-\rho W}\right)=-|\Sigma|^{-1 / 2}\left|\Sigma^{-1}+2 \rho A\right|^{-1 / 2} \exp \left(-\rho\left(c-\frac{1}{2} \rho b^{\prime}\left(\Sigma^{-1}+2 \rho A\right)^{-1} b\right)\right)
$$

Proof. See Danthine and Moresi (1993).

Proof of Corollary 5: First, we want to derive the ex-ante expected utility function for an uninformed trader. Recall that the combination of CARA utility functions and the normality assumption implies that

$$
E\left(U\left(W_{j}^{U}\right) \mid u_{j}, p\right)=-e^{\left(-\frac{1}{\gamma}\left(E\left(W_{j}^{U} \mid u_{j}, p\right)-\frac{1}{2 \gamma} \operatorname{var}\left(W_{j}^{U} \mid u_{j}, p\right)\right)\right)} .
$$

Using the expression of the final wealth of this agent and (10), we have

$$
E\left(U\left(W_{j}^{U}\right) \mid u_{j}, p\right)=-e^{\left(-\left(\frac{1}{\gamma} p u_{j}+\frac{1}{2} \frac{\left(E\left(v \mid u_{j}, p\right)-p\right)^{2}}{\operatorname{var}\left(v \mid u_{j}, p\right)}\right)\right)} .
$$

Let $z_{1}=p-E\left(p \mid u_{j}\right)$, where $E\left(p \mid u_{j}\right)=A_{0}+A_{1} \bar{v}-A_{2}\left(\bar{u}+\frac{\tau_{\eta}}{\tau_{u}+\tau_{\eta}}\left(u_{j}-\bar{u}\right)\right)$. Using the expression of $E\left(v \mid u_{j}, p\right)$ and $\tau^{U}$, the previous conditional expected utility can be written as:

$$
E\left(U\left(W_{j}^{U}\right) \mid u_{j}\right)=-E\left(e^{\left(-\left(c+b z_{1}+A z_{1}^{2}\right)\right)} \mid u_{j}\right),
$$

where

$$
\begin{aligned}
A & =\frac{1}{2} \tau^{U}\left(\frac{1}{\tau^{U}} A_{1} \tau_{\varepsilon} \frac{\tau_{u}+\tau_{\eta}}{A_{2}^{2} \tau_{\varepsilon}+\rho A_{1}^{2}\left(\tau_{u}+\tau_{\eta}\right)}-1\right)^{2} \\
b & =\frac{1}{\gamma} u_{j}+\tau^{U}\left(\frac{A_{1}}{\tau^{U}} \tau_{\varepsilon} \frac{\tau_{u}+\tau_{\eta}}{A_{2}^{2} \tau_{\varepsilon}+\rho A_{1}^{2}\left(\tau_{u}+\tau_{\eta}\right)}-1\right)\left(\bar{v}-E\left(p \mid u_{j}\right)\right), \text { and } \\
c & =u_{j} \frac{1}{\gamma} E\left(p \mid u_{j}\right)+\frac{1}{2} \tau^{U}\left(\bar{v}-E\left(p \mid u_{j}\right)\right)^{2} .
\end{aligned}
$$

Conditional on $u_{j}, z_{1}$ is normally distributed with zero mean and variance

$\Sigma=\operatorname{var}\left(z_{1} \mid u_{j}\right)=A_{1}^{2}\left(\frac{1}{\tau_{v}}+\rho \frac{1}{\tau_{\varepsilon}}\right)+A_{2}^{2} \frac{1}{\left(\tau_{u}+\tau_{\eta}\right)}=\frac{\left(A_{2}^{2} \tau_{\varepsilon}+A_{1}^{2} \rho\left(\tau_{u}+\tau_{\eta}\right)\right) \tau^{U}}{\left(\tau_{u}+\tau_{\eta}\right) \tau_{v} \tau_{\varepsilon}}$.

Since $\Sigma^{-1}+2 A>0$, we can apply the Lemma A.1 and operating, using the expression of $\tau^{U}$, we get

$$
E\left(U\left(W_{j}^{U}\right) \mid u_{j}\right)=-\frac{e^{\left(-\left(\widehat{c}+\widehat{b} u_{j}+\widehat{A} u_{j}^{2}\right)\right)}}{\left(\tau^{U} \frac{\left(\tau_{u}+\tau_{\eta}\right)\left(\tau_{\varepsilon}\left(A_{1}-1\right)^{2}+\rho A_{1}^{2} \tau_{v}\right)+A_{2}^{2} \tau_{v} \tau_{\varepsilon}}{\left(\tau_{u}+\tau_{\eta}\right) \tau_{v} \tau_{\varepsilon}}\right)^{1 / 2}},
$$


where

$$
\begin{aligned}
& \widehat{A}=-\frac{A_{2}^{2} \tau_{\varepsilon}+A_{1}^{2} \rho\left(\tau_{u}+\tau_{\eta}\right)-\tau_{\varepsilon} \tau_{\eta} A_{2} \gamma\left(\frac{\gamma A_{2} \tau_{v} \tau_{\eta}}{\left(\tau_{u}+\tau_{\eta}\right)}+2\left(A_{1}-1\right)\right)}{2\left(\left(\tau_{u}+\tau_{\eta}\right)\left(\tau_{\varepsilon}\left(A_{1}-1\right)^{2}+\rho A_{1}^{2} \tau_{v}\right)+A_{2}^{2} \tau_{v} \tau_{\varepsilon}\right) \gamma^{2}} \\
& \widehat{b}=\frac{\tau_{\varepsilon}\left(A_{2} \bar{u} \frac{\tau_{u}}{\left(\tau_{u}+\tau_{\eta}\right)}-A_{0}\right)\left(\left(\tau_{u}+\tau_{\eta}\right)\left(A_{1}-1\right)+\gamma A_{2} \tau_{v} \tau_{\eta}\right)+\bar{v} \tau_{v}\left(A_{1}^{2} \rho\left(\tau_{u}+\tau_{\eta}\right)-\tau_{\varepsilon} A_{2}\left(\tau_{\eta} \gamma\left(A_{1}-1\right)-A_{2}\right)\right)}{\left(\left(\tau_{u}+\tau_{\eta}\right)\left(\tau_{\varepsilon}\left(A_{1}-1\right)^{2}+\rho A_{1}^{2} \tau_{v}\right)+A_{2}^{2} \tau_{v} \tau_{\varepsilon}\right) \gamma} \\
& \widehat{c}=\frac{\left(\tau_{u}+\tau_{\eta}\right) \tau_{v} \tau_{\varepsilon}\left(\bar{v}-A_{0}-A_{1} \bar{v}+A_{2} \frac{\tau_{u}}{\tau_{u}+\tau_{\eta}} \bar{u}\right)^{2}}{2\left(\left(\tau_{u}+\tau_{\eta}\right)\left(\tau_{\varepsilon}\left(A_{1}-1\right)^{2}+\rho A_{1}^{2} \tau_{v}\right)+A_{2}^{2} \tau_{v} \tau_{\varepsilon}\right)} .
\end{aligned}
$$

Hence, the ex-ante expected utility function for the uninformed trader is given by

$$
E\left(U\left(W_{j}^{U}\right)\right)=-\frac{E\left(e^{\left(-\left(\widehat{c}+\widehat{b} u_{j}+\widehat{A} u_{j}^{2}\right)\right)}\right)}{\left(\tau^{U} \frac{\left(\tau_{u}+\tau_{\eta}\right)\left(\tau_{\varepsilon}\left(A_{1}-1\right)^{2}+\rho A_{1}^{2} \tau_{v}\right)+A_{2}^{2} \tau_{v} \tau_{\varepsilon}}{\left(\tau_{u}+\tau_{\eta}\right) \tau_{v} \tau_{\varepsilon}}\right)^{1 / 2}} .
$$

Performing similar computations as before, we obtain that the ex-ante expected utility function for an informed trader is given by

$$
E\left(U\left(W_{i}^{I}\right)\right)=-\frac{E\left(e^{\left(-\left(\widehat{c}+\widehat{b} u_{i}+\widehat{A} u_{i}^{2}\right)\right)}\right)}{\left(\tau^{I} \frac{\left(\tau_{u}+\tau_{\eta}\right)\left(\tau_{\varepsilon}\left(A_{1}-1\right)^{2}+\rho A_{1}^{2} \tau_{v}\right)+A_{2}^{2} \tau_{v} \tau_{\varepsilon}}{\tau_{\varepsilon} \tau_{v}\left(\tau_{u}+\tau_{\eta}\right)}\right)^{1 / 2}} .
$$

Using the fact that $u_{i}$ and $u_{j}$ are identically distributed, from the previous equality and (14), it follows that $R(\mu)=\left(\frac{\tau^{U}}{\tau^{I}}\right)^{1 / 2}$.

Proof of Proposition 6: Using Corollary 5, the expressions of $\tau^{U}, \tau^{I}$ and the chain rule, we have

$R^{\prime}(\mu)=\frac{1}{2}\left(\frac{\tau^{U}}{\tau^{I}}\right)^{1 / 2} 2 \frac{\left(\tau_{\varepsilon}\left(\tau_{\varepsilon}+\tau_{v} \rho(2-\rho)\right)+\beta^{2} 2 \rho\left(\tau_{u}+\tau_{\eta}\right)(1-\rho)\left(\tau_{\varepsilon}+\rho \tau_{v}\right)\right)\left(\tau_{u}+\tau_{\eta}\right) \beta \tau_{\varepsilon}^{3}}{\left(\beta^{2}\left(\tau_{u}+\tau_{\eta}\right)(1-\rho)\left(\tau_{\varepsilon}+\rho \tau_{v}\right)+\tau_{\varepsilon}\left(\tau_{v}+\tau_{\varepsilon}\right)\right)^{2}\left(\tau_{\varepsilon}+\beta^{2} \rho\left(\tau_{u}+\tau_{\eta}\right)\right)^{2}} \beta^{\prime}(\mu)$.

Since in equilibrium $\beta>0$, it follows that $\operatorname{sign}\left(R^{\prime}(\mu)\right)=\operatorname{sign}\left(\beta^{\prime}(\mu)\right)$. In an equilibrium $\beta-f(\beta)=0$. When the equilibrium is regular, we can apply the Implicit Function Theorem and we get $\frac{\partial \beta}{\partial \mu}=\frac{\frac{\partial}{\partial \mu} f(\beta)}{1-f^{\prime}(\beta)}$. Using the fact that $\frac{\partial f}{\partial \mu}>0$, it follows that $\operatorname{sign}\left(R^{\prime}(\mu)\right)=\operatorname{sign}\left(1-f^{\prime}(\beta)\right)$.

Proof of Corollary 7: Using the expression of $f(\cdot)$, we get that, when $\rho(1-$ $\rho)=0, f(\cdot)$ is a constant function, and obviously, in this case there is one equilibrium and it is stable. Suppose now that $\rho(1-\rho) \neq 0$. In this case $f(\cdot)$ is a decreasing function. Taking into account this fact, and that $f(0)>0$ and $\lim _{\beta \rightarrow \infty}$ $f(\beta)=0$, it follows that the equilibrium is unique. Concerning to stability of the equilibrium notice that, differentiating $f(\beta)$ and evaluating at the fixed point, we 
get $f^{\prime}(\beta)=-\frac{2(1-\rho) \tau_{u} \beta^{2} \rho}{\beta^{2} \rho \tau_{u}(1-\rho)+\tau_{\varepsilon}}<0$. The stability of the equilibrium requires that $-f^{\prime}(\beta)<1$. Direct computations yield that the previous inequality is satisfied if and only if the equilibrium value of $\beta$ satisfies $\beta<\sqrt{\frac{\tau_{\varepsilon}}{(1-\rho) \tau_{u} \rho}}$, or equivalently, $\sqrt{\frac{\tau_{\varepsilon}}{(1-\rho) \tau_{u} \rho}}>f\left(\sqrt{\frac{\tau_{\varepsilon}}{(1-\rho) \tau_{u} \rho}}\right)$, since the function $\beta-f(\beta)$ is increasing, which is equivalent to $\frac{4}{\gamma^{2} \mu^{2} \tau_{u} \tau_{\varepsilon}}>(1-\rho) \rho$.

Proof of Corollary 8: When $\rho=0$, we have

$$
\beta=f(\beta)=\mu \gamma\left(\tau_{\eta} \beta^{2}+\tau_{\varepsilon}\right)+(1-\mu) \gamma \beta^{2} \tau_{\eta},
$$

or equivalently,

$$
P(\beta)=\gamma \tau_{\eta} \beta^{2}-\beta+\gamma \mu \tau_{\varepsilon}=0 .
$$

Notice that $P(\beta)$ is a polynomial of degree 2 in $\beta$. Therefore, we can explicitly compute the roots of this polynomial. If $1<4 \tau_{\varepsilon} \tau_{\eta} \mu \gamma^{2}$, the polynomial $P(\beta)$ has no real roots and, hence, we conclude that a $S L E$ does not exist. Otherwise, that is, if $1 \geq 4 \tau_{\varepsilon} \tau_{\eta} \mu \gamma^{2}$, then $\beta_{1}$ and $\beta_{2}$ are the roots of $P(\beta)$, whose expressions are given in the statement of this corollary.

In relation to stability, straightforward computations yield $0<f^{\prime}\left(\beta_{1}\right)<1$ and $f^{\prime}\left(\beta_{2}\right)>1$. Consequently, the equilibrium with the lowest value of $\beta$ is stable and the other equilibrium is unstable.

Proof of Corollary 9: Note that in this case the fixed-point equation can be written as

$$
\beta=f(\beta)=\mu \Delta \Phi+(1-\mu) \Delta \frac{\beta^{2} \Psi \Phi}{\Phi+\beta^{2}},
$$

where $\Psi=\frac{\tau_{\eta}}{\tau_{u}+\tau_{\eta}}, \Phi=\frac{\tau_{\varepsilon}}{\tau_{u}+\tau_{\eta}}$, and $\Delta=\gamma\left(\tau_{u}+\tau_{\eta}\right)$. Differentiating $f(\beta)$, we get

$$
f^{\prime}(\beta)=(1-\mu) \Delta \frac{2 \Phi^{2} \Psi \beta}{\left(\Phi+\beta^{2}\right)^{2}} .
$$

Notice that if $(1-\mu) \Psi=0$, then the equilibrium is unique since $f(\beta)$ is constant. Suppose that $(1-\mu) \Psi \neq 0$. From the fixed-point equation, we have that in a solution of the fixed-point equation

$$
\Delta=\frac{\beta}{\mu \Phi+(1-\mu) \frac{\beta^{2} \Psi \Phi}{\Phi+\beta^{2}}} .
$$

Substituting this expression in the formula of $f^{\prime}(\beta)$, we get

$$
f^{\prime}(\beta)=(1-\mu) \frac{2 \beta^{2} \Psi \Phi^{2}}{\left(\mu \Phi+(1-\mu) \frac{\beta^{2} \Psi \Phi}{\Phi+\beta^{2}}\right)\left(\Phi+\beta^{2}\right)^{2}} .
$$

We wonder under which conditions there exists a fixed point such that $f^{\prime}(\beta)=1$. Let $z=\beta^{2}$, the previous equality is equivalent to 


$$
z^{2}(\Psi \mu-\mu-\Psi)+z \Phi(\Psi-2 \mu-\Psi \mu)-\Phi^{2} \mu=0 .
$$

The polynomial in the LHS has a discriminant equal to $\Psi \Phi^{2}(1-\mu)(-8 \mu+\Psi-\Psi \mu)$. We distinguish two cases: 1) $-8 \mu+\Psi-\Psi \mu<0$ and 2) $(-8 \mu+\Psi-\Psi \mu) \geq 0$.

- Case 1: $-8 \mu+\Psi-\Psi \mu<0$. In this case the discriminant is negative. Hence,

$z^{2}(\Psi \mu-\mu-\Psi)+z \Phi(\Psi-2 \mu-\Psi \mu)-\Phi^{2} \mu<0$ for all $z$. This implies that in any fixed point $f^{\prime}(\beta)<1$. Hence, we conclude that the equilibrium is unique.

- Case 2: $-8 \mu+\Psi-\Psi \mu \geq 0$. In this case (16) has the following roots:

$$
\begin{aligned}
& \underline{z}=\frac{\Phi(-2 \mu+\Psi-\Psi \mu-\sqrt{\Psi(\mu-1)(8 \mu-\Psi+\Psi \mu})}{2(\Psi+\mu-\Psi \mu)} \text { and } \\
& \bar{z}=\frac{\Phi(-2 \mu+\Psi-\Psi \mu+\sqrt{\Psi(\mu-1)(8 \mu-\Psi+\Psi \mu)})}{2(\Psi+\mu-\Psi \mu)} .
\end{aligned}
$$

Using the fact that $z=\beta^{2}$, substituting the expressions of $\underline{z}$ and $\bar{z}$ in (15), we have three subcases:

- if $-8 \mu+\Psi-\Psi \mu>0$ and $\Delta=\bar{\Delta}$, we have two fixed points, in one of them $\beta=\sqrt{\frac{\Phi(-2 \mu+\Psi-\Psi \mu-\sqrt{\Psi(\mu-1)(8 \mu-\Psi+\Psi \mu)})}{2(\Psi+\mu-\Psi \mu)}}$ and satisfies that $f^{\prime}(\beta)=1$;

- if $-8 \mu+\Psi-\Psi \mu>0$ and $\Delta=\underline{\Delta}$, we have two fixed points, in one of them is $\beta=\sqrt{\frac{\Phi(-2 \mu+\Psi-\Psi \mu+\sqrt{\Psi(\mu-1)(8 \mu-\Psi+\Psi \mu)})}{2(\Psi+\mu-\Psi \mu)}}$ and satisfies that $f^{\prime}(\beta)=1$; and

- if $-8 \mu+\Psi-\Psi \mu=0$, and $\Delta=\underline{\Delta}=\bar{\Delta}=\sqrt{\frac{(\Psi+8)^{2}}{27 \Phi \Psi^{2}}}$, there is a unique fixed point in which $\beta=\sqrt{\frac{1}{3} \Phi}$.

In addition, notice that $\frac{\partial}{\partial \Delta} f(\beta)>0$ and $\frac{\partial}{\partial \Delta} f^{\prime}(\beta)>0$ whenever $(1-\mu) \Psi \neq 0$. Taking into account all these properties and the fact that $\Delta=\gamma\left(\tau_{u}+\tau_{\eta}\right)$, we obtain the stated results of this corollary.

Proof of Corollary 10: In this case the fixed-point equation can be rewritten as

$$
\beta=f(\beta)=\Delta \frac{\left(\Psi \beta^{2}(1-\rho)+\Phi\right) \Phi}{\left(\beta^{2} \rho(1-\rho)+\Phi\right)},
$$

where $\Psi=\frac{\tau_{\eta}}{\tau_{u}+\tau_{\eta}}, \Phi=\frac{\tau_{\varepsilon}}{\tau_{u}+\tau_{\eta}}$, and $\Delta=\gamma\left(\tau_{u}+\tau_{\eta}\right)$. Differentiating $f(\beta)$, we get

$$
f^{\prime}(\beta)=\Delta \Phi\left(-\frac{2(\rho-\Psi)(1-\rho) \Phi \beta}{\left(\beta^{2} \rho(1-\rho)+\Phi\right)^{2}}\right) .
$$


Notice that if $(\rho-\Psi)(1-\rho) \geq 0$, then we get uniqueness of the equilibrium since the function $f(\cdot)$ is decreasing. Suppose now that $(\rho-\Psi)(1-\rho)<0$. From the fixed-point equation, we have that in an equilibrium

$$
\Delta=\frac{\beta\left(\beta^{2} \rho(1-\rho)+\Phi\right)}{\Phi\left(\Psi \beta^{2}(1-\rho)+\Phi\right)} .
$$

Substituting this expression in the formula of $f^{\prime}(\beta)$, we get

$$
f^{\prime}(\beta)=\frac{\beta^{2}}{\left(\Psi \beta^{2}(1-\rho)+\Phi\right)}\left(-\frac{2(\rho-\Psi)(1-\rho) \Phi}{\left(\beta^{2} \rho(1-\rho)+\Phi\right)}\right) .
$$

We wonder under which conditions there exists a fixed point such that $f^{\prime}(\beta)=1$ Let $z=\beta^{2}$, the previous equality is equivalent to

$$
z \Phi(1-\rho)(\Psi-3 \rho)-\Phi^{2}-z^{2} \Psi \rho(\rho-1)^{2}=0 .
$$

The polynomial in the LHS has a discriminant equal to $\Phi^{2}(\rho-1)^{2}(\Psi-9 \rho)(\Psi-\rho)$. We distinguish two cases: 1) $\Psi-9 \rho<0$, and 2) $\Psi-9 \rho \geq 0$.

- Case 1: $\Psi-9 \rho<0$. In this case the discriminant is negative. Hence, $z \Phi(1-\rho)(\Psi-3 \rho)-\Phi^{2}-z^{2} \Psi \rho(\rho-1)^{2}<0$ for all $z$. This implies that in any fixed point $f^{\prime}(\beta)<1$. Consequently, the equilibrium is unique.

- Case 2: $\Psi-9 \rho \geq 0$. In this case (18) has the following roots:

$$
\begin{aligned}
& \underline{z}=\frac{\Phi((\Psi-3 \rho)-\sqrt{(\Psi-9 \rho)(\Psi-\rho)})}{2 \Psi \rho(1-\rho)} \text { and } \\
& \bar{z}=\frac{\Phi((\Psi-3 \rho)+\sqrt{(\Psi-9 \rho)(\Psi-\rho)})}{2 \Psi \rho(1-\rho)}
\end{aligned}
$$

Using the fact that $z=\beta^{2}$, substituting the expressions of $\underline{z}$ and $\bar{z}$ in (17), we have three subcases:

- if $\Psi-9 \rho>0$ and $\Delta=\bar{\Delta}=\sqrt{\frac{\left(\left(18 \rho \Psi-27 \rho^{2}+\Psi^{2}\right)+(\Psi-9 \rho) \sqrt{(\Psi-9 \rho)(\Psi-\rho)}\right)}{8 \Psi(1-\rho) \Psi^{2} \Phi}}$, there are two fixed points, in one of them $\beta=\sqrt{\frac{\Phi((\Psi-3 \rho)-\sqrt{(\Psi-9 \rho)(\Psi-\rho)})}{2 \Psi \rho(1-\rho)}}$ and satisfies that $f^{\prime}(\beta)=1$,

- if $\Psi-9 \rho>0$ and $\Delta=\underline{\Delta}=\sqrt{\frac{\left(\left(18 \rho \Psi-27 \rho^{2}+\Psi^{2}\right)-(\Psi-9 \rho) \sqrt{(\Psi-9 \rho)(\Psi-\rho)}\right)}{8 \Psi(1-\rho) \Psi^{2} \Phi}}$, there are two fixed points, in one of them $\beta=\sqrt{\frac{\Phi((\Psi-3 \rho)+\sqrt{(\Psi-9 \rho)(\Psi-\rho)})}{2 \Psi \rho(1-\rho)}}$ and satisfies that $f^{\prime}(\beta)=1$, and

- if $\Psi-9 \rho=0$ and $\Delta=\underline{\Delta}=\bar{\Delta}=\sqrt{\frac{1}{3 \Phi \Psi\left(-\frac{1}{9} \Psi+1\right)}}$, we have a unique fixed point. 
Notice that $\frac{\partial}{\partial \Delta} f(\beta)>0$ and $\frac{\partial}{\partial \Delta} f^{\prime}(\beta)>0$ whenever $(\rho-\Psi)(1-\rho)<0$. Taking into account all these properties and the fact that $\Delta=\gamma\left(\tau_{u}+\tau_{\eta}\right)$, we obtain the stated results of this corollary.

Proof of Corollary 11: When $\mu=1$,

$$
\begin{aligned}
f^{\prime}(\beta) & =\Delta \Phi\left(-\frac{2(\rho-\Psi)(1-\rho) \Phi \beta}{\left(\beta^{2} \rho(1-\rho)+\Phi\right)^{2}}\right) \text { and } \\
f^{\prime \prime}(\beta) & =\Delta \Phi^{2} \frac{2(\rho-\Psi)(1-\rho)\left(3 \rho(1-\rho) \beta^{2}-\Phi\right)}{\left(\beta^{2} \rho(1-\rho)+\Phi\right)^{3}} .
\end{aligned}
$$

Note that if $\rho \leq \Psi$, then the function $f(\cdot)$ has an increasing S-shape. Combining this result with the fact $f(0)>0$ and $\lim _{\beta \rightarrow \infty} f(\beta)<\infty$, we get three possibilities:

1 ) in case of uniqueness of the $S L E$, generically $0 \leq f^{\prime}(\beta)<1$ at the equilibrium, which implies that the equilibrium is stable.

2 ) in case of two $S L E, 0<f^{\prime}(\beta)<1$ in one equilibrium and $f^{\prime}(\beta)=1$ in the other equilibrium. This implies that there is one stable equilibrium and one unstable equilibrium.

$3)$ in case of three $S L E, 0<f^{\prime}(\beta)<1$ in the two equilibria with extreme values of $\beta$, whereas $f^{\prime}(\beta)>1$ in the equilibrium with the intermediate value of $\beta$. Hence, the two first equilibria are stable and the last one is unstable.

Suppose now that $\rho>\Psi$. In this case $f^{\prime}(\beta)<0$, and therefore, there is a unique equilibrium. The stability of the fixed point requires that $-f^{\prime}(\beta)<1$. Direct computations yield that this inequality is equivalent to

$$
k(\beta)>0,
$$

where $k(b)=\Phi^{2}+b^{4} \rho \Psi(\rho-1)^{2}+b^{2} \Phi(1-\rho)(-\rho+3 \Psi)$. We distinguish 3 cases: 1) $\Psi<\rho<9 \Psi$, 2) $\rho=9 \Psi$, and 3) $1>\rho>9 \Psi$.

- Case 1: $\Psi<\rho<9 \Psi$. In this case the polynomial $k(x)$, where $x=b^{2}$, has a negative discriminant and, consequently, it does not have any real root. In all values the polynomial is positive, and in particular, in the fixed point. This implies that (19) holds. Hence, we get that the equilibrium is stable.

- Case 2: $\rho=9 \Psi$. In this case there is a unique positive root of $k(b)$, $b=\sqrt{\frac{\Phi}{3 \Psi(-9 \Psi+1)}}$. In addition, notice that $f\left(\sqrt{\frac{\Phi}{3 \Psi(-9 \Psi+1)}}\right)=\frac{1}{3} \Phi \Delta \neq$ $\sqrt{\frac{\Phi}{3 \Psi(-9 \Psi+1)}}$ a. s.. This implies that (19) holds a.s.. Hence, we get that the equilibrium is stable a.s..

- Case 3: $1>\rho>9 \Psi$. In this case, the positive roots of $k(b)$ are

$$
\begin{aligned}
& \underline{b}^{2}=\frac{\Phi}{2 \rho \Psi(1-\rho)}(\rho-3 \Psi-\sqrt{(\rho-9 \Psi)(\rho-\Psi)}) \text { and } \\
& \bar{b}^{2}=\frac{\Phi}{2 \rho \Psi(1-\rho)}(\rho-3 \Psi+\sqrt{(\rho-9 \Psi)(\rho-\Psi)}) .
\end{aligned}
$$


The condition (19) is satisfied whenever the fixed point satisfies $\beta<\underline{b}$ or $\beta>\bar{b}$. Since the function $\beta-f(\beta)$ is strictly increasing, the previous inequalities hold if and only if $\underline{b}>f(\underline{b})$ or $\bar{b}<f(\bar{b})$. Moreover, the inequality $\underline{b}>f(\underline{b})$ is equivalent to $\underline{g}(\Delta)>1$, where

$$
\underline{g}(\Delta)=\frac{\rho\left(12 \Psi^{2}-9 \Psi \rho+\rho^{2}+(4 \Psi-\rho)(\sqrt{(\rho-9 \Psi)(\rho-\Psi)})\right)}{\Psi^{3}(1-\rho)(-9 \Psi+5 \rho-3 \sqrt{(\rho-9 \Psi)(\rho-\Psi)}) \Delta^{2} \Phi} .
$$

On the other hand, the inequality $\bar{b}<f(\bar{b})$ is equivalent to $\bar{g}(\Delta)<1$, where $\bar{g}(\Delta)=\frac{\rho\left(12 \Psi^{2}-9 \Psi \rho+\rho^{2}+(\rho-4 \Psi) \sqrt{9(\rho-9 \Psi)(\rho-\Psi)}\right)}{\Psi^{3}(1-\rho)(-9 \Psi+5 \rho+3 \sqrt{(\rho-9 \Psi)(\rho-\Psi)}) \Delta^{2} \Phi}$. Some algebra tells us that $\underline{g}(\Delta)$ and $\bar{g}(\Delta)$ are decreasing functions in $\Delta, \underline{g}(\Delta)<\bar{g}(\Delta), \lim _{\Delta \rightarrow 0}$ $\underline{g}(\Delta)=\lim _{\Delta \rightarrow 0} \bar{g}(\Delta)=\infty$ and $\lim _{\Delta \rightarrow \infty} \underline{g}(\Delta)=\lim _{\Delta \rightarrow \infty} \bar{g}(\Delta)=0$. Let $\Delta_{1}$ and $\Delta_{2}$ such that $\underline{g}\left(\Delta_{1}\right)=1$ and $\bar{g}\left(\Delta_{2}\right)=1$. Direct computations yield

$$
\begin{aligned}
& \Delta_{1}=\sqrt{\frac{\rho\left(12 \Psi^{2}-9 \Psi \rho+\rho^{2}+(4 \Psi-\rho)(\sqrt{(\rho-9 \Psi)(\rho-\Psi)})\right.}{\Psi^{3}(1-\rho)(-9 \Psi+5 \rho-3 \sqrt{(\rho-9 \Psi)(\rho-\Psi)}) \Phi}} \text { and } \\
& \Delta_{2}=\sqrt{\frac{\rho\left(12 \Psi^{2}-9 \Psi \rho+\rho^{2}+(\rho-4 \Psi) \sqrt{(\rho-9 \Psi)(\rho-\Psi)}\right.}{\Psi^{3}(1-\rho)(-9 \Psi+5 \rho+3 \sqrt{(\rho-9 \Psi)(\rho-\Psi)}) \Phi}} .
\end{aligned}
$$

Then, taking into account that $\Delta=\gamma\left(\tau_{u}+\tau_{\eta}\right)$, we obtain

- for all $\gamma<\gamma_{1}=\frac{\Delta_{1}}{\tau_{u}+\tau_{\eta}}, 1<\underline{g}(\Delta)<\bar{g}(\Delta)$, and hence, we conclude that the equilibrium is stable,

- for all $\gamma>\gamma_{2}=\frac{\Delta_{2}}{\tau_{u}+\tau_{\eta}}, \underline{g}(\Delta)<\bar{g}(\Delta)<1$, and hence, we conclude that the equilibrium is stable, and

- for all $\gamma \in\left[\gamma_{1}, \gamma_{2}\right], g(\Delta) \leq 1 \leq \bar{g}(\Delta)$, and hence, we conclude that the equilibrium is unstable.

\section{References}

[1] Admati, A. R., 1985. A noisy rational expectations equilibrium for multiasset securities markets. Econometrica 53, 629-657.

[2] Amador, M., Weill, P.O., 2010. Learning from prices: Public communication and welfare. Mimeo.

[3] Barlevy, G., Veronesi P., 2000. Information acquisition in financial markets. Review of Economic Studies 67, 79-90. 
[4] Barlevy, G., Veronesi P., 2008. Information acquisition in financial markets: a correction. Mimeo.

[5] Blume, L. E., Bray, M. M., Easley, D., 1982. Introduction to the stability of rational expectations equilibrium. Journal of Economic Theory 26, 313-317.

[6] Chamley, C., 2007. Complementarities in Information Acquisition with Short-Term Trades. Theoretical Economics, 2, 441-467.

[7] Chamley, C., 2008a. On acquisition of information in financial markets. Review of Economic Studies 75, 1081-1084.

[8] Chamley, C., 2008b. Comment on equilibria on Ganguli and Yang (2006, 2007). Mimeo, Boston University.

[9] Danthine, J. P., Moresi, S., 1993. Volatility, information, and noise trading. European Economic Review 37, 961-982.

[10] Diamond, D. W., Verrecchia, R. E., 1981. Information aggregation in a noisy rational expectations economy. Journal of Financial Economics 9, 221-235.

[11] Echenique, F., 2002. Comparative statics by adaptive dynamics and the Correspondence Principle. Econometrica 70 (2), 833-844.

[12] Ganguli, J. V., Yang L., 2009. Complementarities, multiplicity, and supply information. Journal of the European Economic Association 7, 90-115.

[13] Gennote, G., Leland, H., 1990. Market liquidity, hedging, and crashes. American Economic Review 80 (5), 999-1021.

[14] Goldstein, I., Ozdenoren E., Yuan,K., 2010. Learning and complementarities in speculative attacks. Review of Economic Studies, forthcoming.

[15] Grossman, S., Stiglitz, J., 1980. On the impossibility of informationally efficient markets. American Economic Review 70, 393-408.

[16] Grundy, B., McNichols, M., 1989. Trade and revelation of information through prices and direct disclosure. Review of Financial Studies 2, 495526.

[17] Guesnerie, R., 2002. Anchoring economic predictions in common knowledge. Econometrica 70, 439-480.

[18] Hatchondo, J. C., Krusell, P., Schneider, M., 2010. Asset trading and valuation with uncertain exposure. Mimeo, Standford University.

[19] He, H., Wang, J., 1995. Differential information and dynamic behavior of stock trading volume. Review of Financial Studies 8, 919-972. 
[20] Heinemann, M., 2010. Stability under learning of equilibria in financial markets with supply information. Economics Bulletin 30 (1), 383-391.

[21] Hellwig, M. F., 1980. On the efficiency of competitive stock markets where traders have diverse information. Journal of Economic Theory 22, 477-498.

[22] Hellwig, C., Veldkamp, L., 2009. Knowing what others know: Coordination motives in information acquisition. Review of Economic Studies 76, 223251.

[23] Hirshleifer, D., Subrahmanyam, A., Titman, S., 1994. Security analysis and trading patterns when some investors receive information before others. Journal of Finance, 49, 1665-1698.

[24] Lundholm, R. J., 1988. Price-signal relations in the presence of correlated public and private information. Journal of Accounting Research 26, 107118.

[25] McCafferty, S., Driskill, R., 1980. Problems of existence and uniqueness in nonlinear rational expectations models. Econometrica 48, 1313-1317.

[26] Medrano, L., Vives, X., 2004. Regulating insider trading when investment matters? Review of Finance 8, 199-277.

[27] Veldkamp, L., 2006a. Information markets and the comovement of asset prices. Review of Economic Studies. 73, 823-845.

[28] Veldkamp, L., 2006b. Media frenzies in markets for financial information. American Economic Review, 96, 3, 577-601.

[29] Vives, X., 1990. Nash equilibrium with strategic complementarities. Journal of Mathematical Economics 19 (3), 305-321.

[30] Vives, X., 1993. How fast do rational agents learn? Review of Economic Studies 60, 329-347.

[31] Vives, X., 1995. Short term investment and the informational efficiency of the market. Review of Financial Studies 8, 125-160.

[32] Vives, X., 2008. Information and Learning in Markets, Princeton University Press, Princeton, N. J. 